\title{
Solution-processable triarylamine-based high-performance polymers for resistive switching memory devices
}

\author{
Hung-Ju Yen ${ }^{1}$ and Guey-Sheng Liou ${ }^{2}$ \\ This review summarizes the most widely used mechanisms in high-performance polymeric resistive memory devices, such as \\ charge transfer, space charge trapping and filament conduction. In addition, recent studies of functional high-performance \\ polymers for memory device applications are reviewed, compared and differentiated based on the mechanisms and structural \\ design methods used. By carefully designing the polymeric structure based on these systematically investigated switching \\ mechanisms, almost all types of current memory characteristics can be reproduced, and these memory properties show extremely \\ high endurance during long-term operation, which makes polyimides very suitable materials for memory applications.
}

Polymer Journal (2016) 48, 117-138; doi:10.1038/pj.2015.87; published online 7 October 2015

\section{INTRODUCTION}

High-performance polymers

Today, life without polymers is unimaginable. Polymers have become the major synthetic materials of the 21st century. High-performance polymers (HPPs) are particularly desirable. The synthesis and development of HPPs over the past 30 years have drawn the attention of many polymer scientists and investigators. These polymers generally possess excellent physical deformation resistance and chemical deterioration resistance at high temperatures over long periods of time. The quest for HPPs began in the late 1950s to meet the demands of the military, aerospace, machine-building and electronics industries, among many other industrial applications.

Hill and Walker ${ }^{1}$ first noted that the incorporation of aromatic segments into a polymer generally results in a notable increase in its thermal stability. For this reason, much of the research work has been directed toward aromatic compositions. Hence, HPPs usually tend to contain large numbers of aromatic units in their structures. Several of these aromatic HPPs have been used for commercial applications, such as aromatic polyamides, polyimides, polyesters, polysulfones, polytriphenylamine and heterocyclic polymers (Scheme 1). Aromatic polyamides (aramids) and polyimides, such as DuPont's Kevlar fiber and Kapton film, respectively, have been well known for a long time and constantly attract more interest than other HPPs for their useful properties, such as excellent thermal and oxidative stabilities, high mechanical strengths, low flammabilities and good chemical and radiation resistances. ${ }^{2-13}$

However, the rigidity of the backbone and strong hydrogen bonding of these HPPs result in high melting or glass-transition temperatures $\left(T_{\mathrm{g}}\right)$ and limited solubility in most organic solvents. ${ }^{12,14}$ These properties make them difficult to process, thus restricting their technological and practical applications. To overcome such a dilemma without sacrificing high thermal stability, the introduction of bulky and packing-disruptive groups (for example, triphenylamine (TPA) derivatives) into the polymer backbone is a feasible approach. ${ }^{15-22}$ Since 2002, we have developed processes for the synthesis of soluble aromatic polyimides and polyamides containing TPA units. Because of the incorporation of the bulky, propeller-shaped TPA structure along the polymer backbone, all of the polymers were amorphous with good solubilities, good film-forming capabilities and high thermal stabilities. $^{23-25}$

Polyimide. Aromatic polyimides can be categorized as an important class of materials because of their many desirable characteristics and often replace glass and metals in industrial applications, especially in the semiconductor and electric packaging industries. The outstanding properties of aromatic polyimides come from their rigid backbones and the strong intramolecular and intermolecular forces between the polymer chains, which result from charge transfer (CT) complex formation between electron-donating (diamine moiety) and electronaccepting (dianhydride moiety) segments.

TPA-based polyimides were first prepared in 1992 from 4,4'diaminotriphenylamine and various tetracarboxylic dianhydrides by Imai and colleagues (Scheme 2). ${ }^{26,27}$ These polymers were soluble in many polar solvents and showed useful levels of thermal stability associated with high glass-transition temperatures $\left(T_{\mathrm{g}}\right)\left(287-331^{\circ} \mathrm{C}\right)$ and high char yields.

\footnotetext{
${ }^{1}$ Los Alamos National Laboratory, Physical Chemistry and Applied Spectroscopy (C-PCS), Chemistry Division, Los Alamos, NM, USA and ${ }^{2}$ Functional Polymeric Materials Laboratory, Institute of Polymer Science and Engineering, National Taiwan University, Taipei, Taiwan

Correspondence: Professor G-S Liou, Functional Polymeric Materials Laboratory, Institute of Polymer Science and Engineering, National Taiwan University, 1 Roosevelt Road, 4th Section, Taipei 10617, Taiwan.

E-mail: gsliou@ntu.edu.tw

Received 13 July 2015; revised 8 August 2015; accepted 10 August 2015; published online 7 October 2015
} 


\section{Aromatic polyamide}

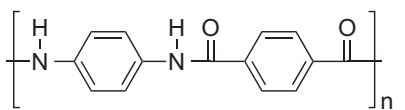

Kevlar

Aromatic polyimide<smiles>CCn1c(=O)c2cc3c(=O)n(-c4ccc(Oc5ccc(C)cc5)cc4)c(=O)c3cc2c1=O</smiles>

Kapton

Aromatic polyester

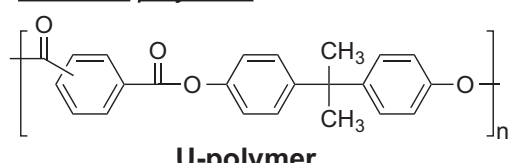

U-polymer

Aromatic heterocyclic polymer<smiles></smiles>

PBT<smiles>CCCCC(=O)c1cccc(C(=O)Nc2cccc(NC(C)C)c2)c1</smiles>

Nomex
Upilex R

\section{Aromatic polysulfone}<smiles>CC(C)(C)c1ccc(S(=O)(=O)c2ccc(Oc3ccc(C(C)(C)c4ccc(OC5CCCC5)cc4)cc3)cc2)cc1</smiles>

Aromatic polytriphenylamine<smiles>[X]c1ccc(N(c2ccc(C)cc2)c2ccc(C)cc2)cc1</smiles>

Scheme 1 Chemical structures of the high-performance polymers.
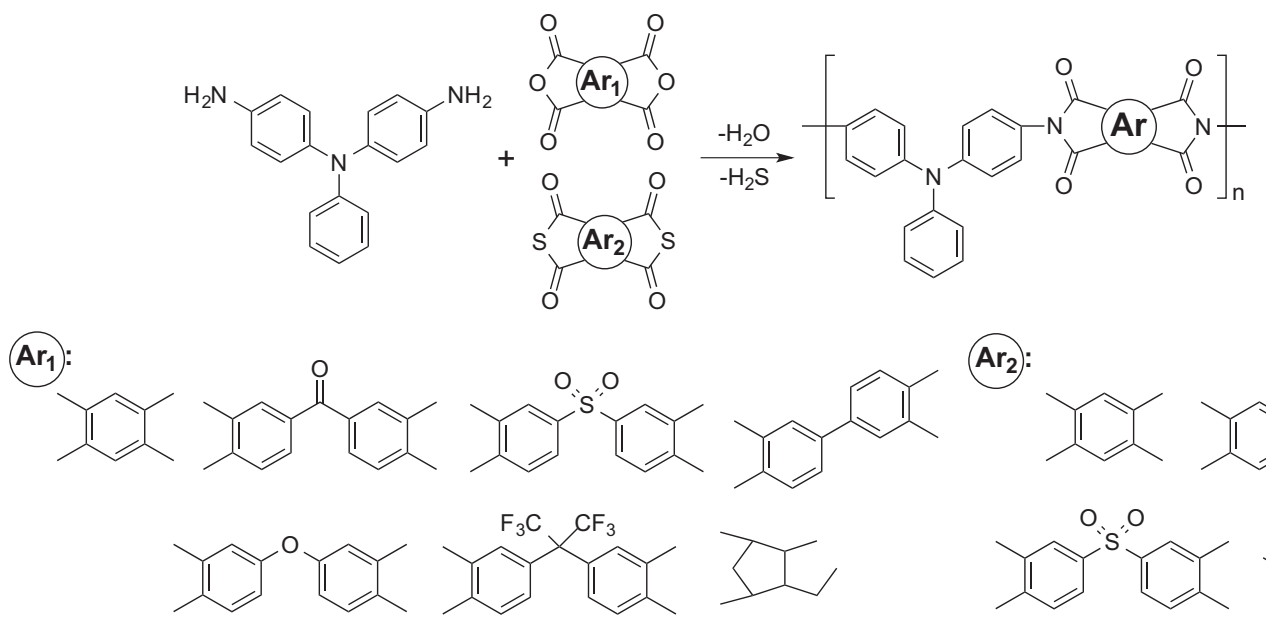

$\mathrm{Ar}_{2}$ :<smiles>Cc1cc(C)c(C)cc1C</smiles>

Scheme 2 Chemical structures of triphenylamine-based polyimides.

Polyamide. It is well known that the aromatic polyamides have attracted great interest as microelectronic materials owing to their outstanding thermal and mechanical resistances. ${ }^{2}$ Incorporation of packing-disruptive TPA units into polyamides preserves their high thermal stabilities, glass transition temperatures and solubilities, enhancing their film-forming abilities, which is beneficial for the fabrication of large-area, thin-film electrochromic devices. In addition, this change provides electroactive centers that facilitate both processing and electrochromic applications.
Polyamides containing TPA groups were first prepared in 1990 from 4,4'-diaminotriphenylamine and various dicarboxylic acids by Imai and colleagues via phosphorylation polyamidation (Scheme 3). ${ }^{28}$

Polytriphenylamine. In addition to the polyimides and polyamides prepared by polycondensation reactions, polymeric TPAs can be prepared using Grignard $^{29}$ and palladium coupling reactions ${ }^{30}$ (Scheme 4). However, these synthetic methods are not straightforward and require multiple steps to prepare the corresponding monomers. 

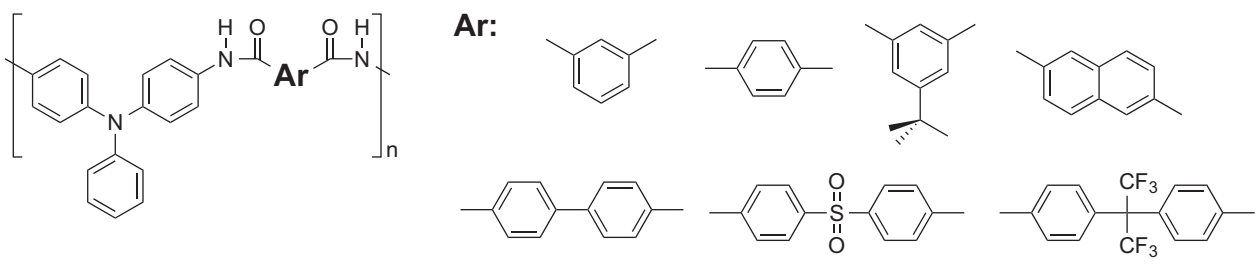

Scheme 3 Chemical structures of triphenylamine-based polyamides.<smiles>[R]c1ccc(N(c2ccc(Br)cc2)c2ccc(Br)cc2)cc1</smiles>

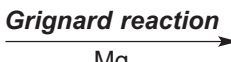<smiles>[R]c1ccc(N(c2ccc(C)cc2)c2ccc(C(C)(C)C)cc2)cc1</smiles><smiles>C=CC(C)c1ccc(Nc2ccc(-c3ccc(Nc4ccc(C(C)CC)cc4)cc3)cc2)cc1</smiles><smiles>Brc1ccc(Br)cc1</smiles><smiles>CCC(C)c1ccc(N(c2ccc(-c3ccc(N(c4ccc(C(C)C)cc4)C(C)(C)C)cc3)cc2)c2ccc(C(C)C)cc2)cc1</smiles><smiles>[X]c1ccc(N(c2ccccc2)c2ccccc2)cc1</smiles>

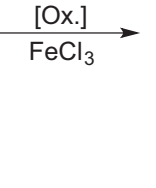<smiles>[X]c1ccc(N(c2ccc(C)cc2)c2ccc(C(C)(C)C)cc2)cc1</smiles>

X: $-\mathrm{CH}_{3},-\mathrm{OCH}_{3},-\mathrm{CN},-\mathrm{NO}_{2}$,

Scheme 4 Chemical structures of polytriphenylamines.

Therefore, we need a simple and rapid method to replace the aforementioned reaction.

A series of different para-substituted TPA-based polymers with high molecular weights were prepared by oxidative coupling polymerization using $\mathrm{FeCl}_{3}$ as an oxidant. ${ }^{31,32}$ These results demonstrated that incorporating electron-withdrawing substituents at the para-position of TPA could not only increase the molecular weight of the poly (triphenylamine)s while maintaining good thermal stability but also yield adjustable band gaps and good electrochemical stabilities for optoelectronic applications.

\section{Resistive memory devices}

Devices incorporating switchable resistive materials are generically classified as resistor-type memory or resistive random access memory. Unlike transistor and capacitor memories, a resistor-type memory does not require a specific cell structure (for example, field-effect transistor (FET)) or to be integrated with the complementary metaloxide-semiconductor technology.

Resistor-type memories can store data by defining the two states of electrical bistability (high and low conductivity) as ' 1 ' and ' 0 ', usually through changes in the intrinsic properties of materials, such as CT, phase change, conformation change and reduction-oxidation (redox) state, that occur in response to the applied voltage or electric field. For this type of resistive memory, the design and synthesis of resistive memory materials are very important. Organic small-molecule compounds, polymers, inorganic materials and inorganic/organic hybrid materials have been successfully applied as memory materials in resistive memory devices.

Owing to the advantages of good scalability, flexibility, low cost, ease of processing, 3D-stacking capability and large capacity for data storage, polymer-based resistive memories are a promising alternative to conventional inorganic semiconductor-based memory technology. Extensive research into new polymer materials and device structures has resulted in good memory performances, such as a large ON-OFF current ratio, low operation voltage, relatively long retention time and high operation endurance. ${ }^{33}$

\section{MECHANISM}

Many studies have been conducted to understand the electric switching phenomena of memory devices. Although this field is still controversial, researchers have proposed several well-established switching mechanisms based on theoretical simulations, experimental results and advanced analytical techniques. ${ }^{33-41}$ In this review, we summarize the mechanisms that are used most widely in HPP resistive 
memory devices, including CT, space charge trapping and filamentary conduction.

\section{Charge transfer}

CT can be described as the partial transfer of electronic charge from the donor (D) to the acceptor (A) moiety in an electron D-A system induced by applying a suitable voltage, which can result in a sharp increase in conductivity. ${ }^{42}$ To obtain a better understanding of switching mechanisms, several methods, such as density functional theory (DFT) calculations, UV/vis absorption spectroscopy, in situ fluorescence spectroscopy and transmission electron microscopy, have been used to investigate and explain the CT phenomenon. ${ }^{43-46} \mathrm{CT}$ is anticipated to occur most frequently in polymers with D-A structures. ${ }^{47-49}$ Memory behaviors based on D-A polymers can be adequately tuned through the modification of the polymer structures.
By tuning the electron-donating or electron-accepting capabilities of D-A polymers, different memory behaviors can be achieved. ${ }^{50} \mathrm{~A}$ strong dipole moment in a polymer is also beneficial to maintain the conductive CT state, usually leading to non-volatile behavior. If the dipole moment is not strong enough, then the conductive CT state is not stable after removal of the electric field and a volatile memory characteristic will be observed.

Conformational change. Conformational change is also a widely used mechanism in polymer resistive memory devices. To investigate the linkage effect between a TPA donor and a hexafluoroisopropyl bis (phthalic dianhydride) (6FDA) acceptor, Ueda and colleagues ${ }^{51}$ reported two PIs, $\mathbf{P} \mathbf{1}$ and $\mathbf{P} 2$, consisting of electron-donating AAPT (4-amino- $4^{\prime}$-( $p$-aminophenoxy)triphenylamine) and APT $\left(4,4^{\prime}\right.$-bis $(p$ aminophenoxy)triphenylamine), respectively (Scheme 5). Memory

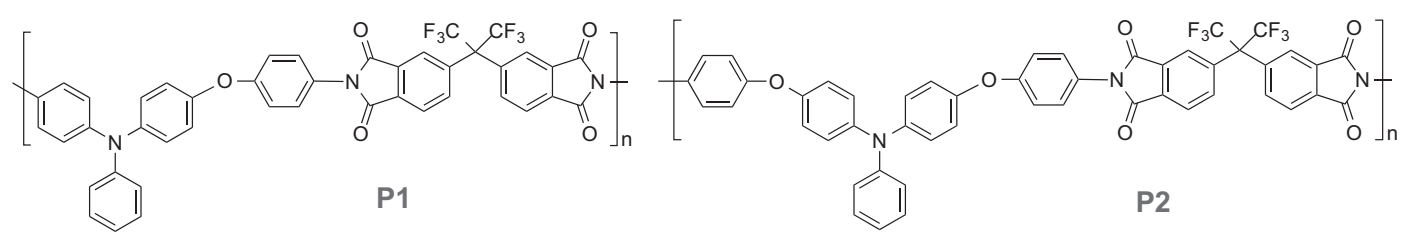<smiles>COc1ccc(N(c2ccc(N3C(=O)c4ccc(C(F)(F)F)cc4C3=O)cc2)c2ccc(N(c3ccc(OC)cc3)c3ccc(C(F)(F)F)cc3)cc2)cc1</smiles><smiles>CNC(C)NCCCCC(=O)c1ccc(C(C)(F)c2ccc(C(=O)Nc3ccc(N(c4ccc(OC)cc4)c4ccc(N(c5ccc(C)cc5)c5ccc(OC)cc5)cc4)cc3)cc2)cc1</smiles>

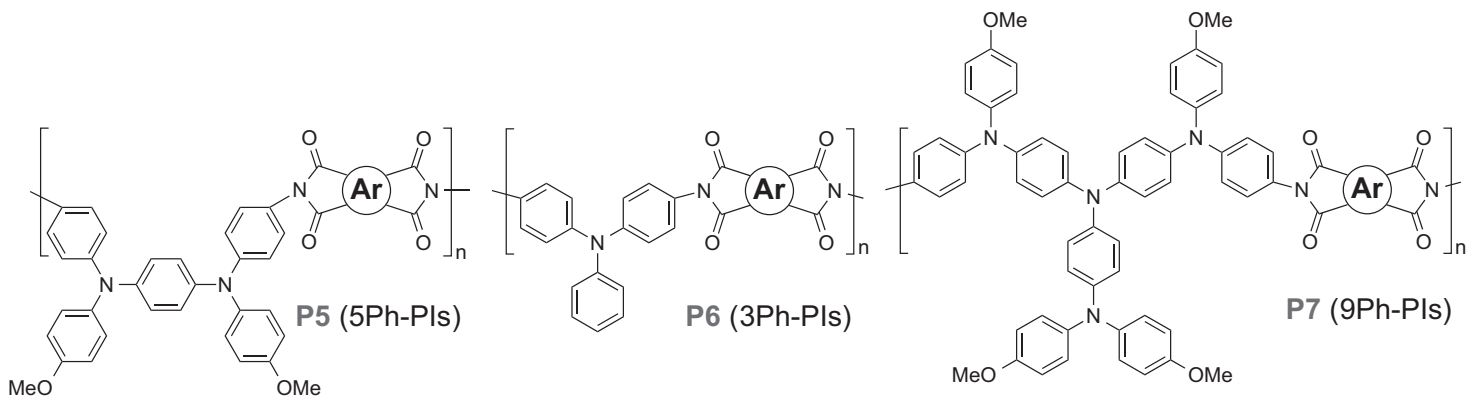<smiles>Cc1ccc(Oc2ccc(C)c(Cc3ccc(C(c4ccc(C)c(C)c4)(c4ccc(C)c(Cc5cc([SH](=O)(O)c6ccc(C)c(C)c6)ccc5C)c4)C(F)(F)F)c(C)c3)c2)cc1C</smiles>

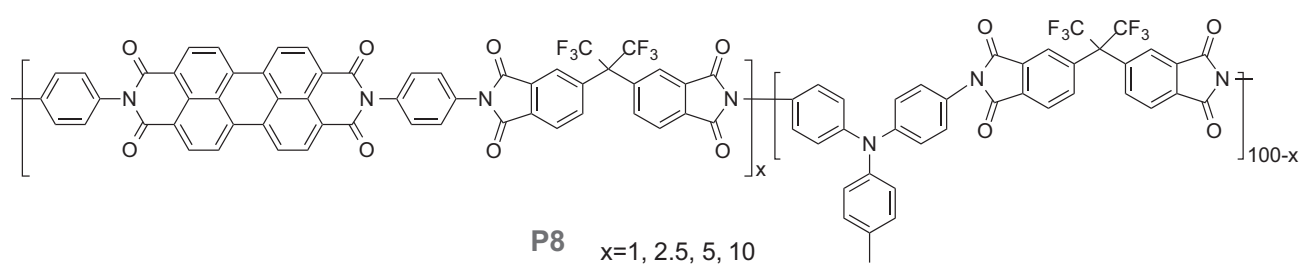

Scheme 5 Chemical structures of some D-A type polymers with charge transfer characteristics. A full color version of this scheme is available at Polymer Journal online. 
devices based on P1 and P2 exhibited dynamic random access memory (DRAM) and static random access memory (SRAM) behaviors, respectively, mainly due to differences in linkage conformation. Theoretical calculations showed that the dual-mediated phenoxy linkage of $\mathbf{P 2}$ would lead to a more twisted conformation than the mono-substituted P1. Thus, a potential barrier would be produced for P2 that would delay the back CT process induced by the electric field, resulting in the SRAM behavior.

In another study, ${ }^{52}$ we prepared the functional PI P3 and polyamide P4 consisting of electron-donating $N, N^{\prime}$-bis(4-aminophenyl)- $N, N^{\prime}$-di (4-methoxylphenyl)-1,4-phenylenediamine for memory application, and the corresponding memory devices exhibited DRAM and SRAM behaviors, respectively. The phthalimide moieties of $\mathbf{P} 3$ form a new plane (dihedral angles $179.5^{\circ}$ ) with adjacent phenyl rings that favor the occurrence of back CT; however, the conformation of $\mathrm{N}$-phenylbenzamide in P4 is not a planar structure, which may block back CT, resulting in a longer retention time in the polyamide system after removal of the applied power (SRAM).

LUMO energy level. The LUMO energy also has an important role in stabilizing the CT state. Because the CT state is a meta-stable state, lower LUMO energy levels provide a more stable CT state. Our group reported a series of functional PIs $\mathbf{P} 5^{48}$ with different dianhydrides, including 6FDA, oxydiphthalic dianhydride, DSDA (3,3',4,4'-diphenylsulfonetetracarboxylic dianhydride), PMDA (pyromellitic dianhydride) and NPDA (1,4,5,8-naphthalenetetracarboxylic dianhydride). As the electron-withdrawing capability of the PIs increases (the LUMO energy level decreases), the retention time of the corresponding memory device increases, causing the memory properties to vary from DRAM to SRAM and write-once read-many times (WORM).

HOMO energy level. The HOMO energy level is another important issue for the CT states. In a recent study, ${ }^{53}$ we prepared and studied memory devices derived from 3Ph-PIs (P6), 5Ph-PIs (P5) and 9Ph-PIs (P7) with different electron-donation abilities. As the electron donating ability increases from $\mathbf{P 6}$ through $\mathbf{P 5}$ to P7, the retention time of the memory device exhibits a systematic increase. For example, PIs with the 6FDA dianhydride showed only DRAM behavior for P6-6FPI and P5-6FPI but SRAM behavior for P7-6FPI. Therefore, facile changes in chemical structure can yield tunable memory behaviors with different retention times.

Dipole moment. An interesting exception to the effects of energy levels was also reported by our group. ${ }^{48}$ Although P5-DSPI has a higher LUMO energy level than P5-PMPI, it showed only non-volatile WORM behavior instead of the volatile properties. This mismatch between LUMO energy and memory characteristics could be explained from the viewpoint of the dipole moment. The main difference between these two PIs is that P5-DSPI has a higher dipole moment (5.45 D) than P5-PMPI (3.70 D). Accordingly, this higher dipole moment resulted in non-volatile memory properties due to the formation of more stable CT states.

Large conjugation. Introducing a large conjugated or highly electronaffinitive unit is a simple and effective approach for developing PIs with non-volatile memory behavior. ${ }^{54}$ For example, the introduction of a small amount ( $5 \mathrm{~mol} \%$ ) of perylene bisimide dramatically changes the memory properties of $\mathbf{P 8}$ from volatile DRAM to the non-volatile WORM type, possibly owing to stabilization of the separated charge by the large conjugation and trapping by the deep LUMO energy level arising from the highly electron-affinitive perylene bisimide.
Space charge traps

When the interface between the electrode and the polymer is ohmic and the polymer is trap-free, carriers near the electrode accumulate and build up a space charge region. Mutual repulsion between individual charges restricts the total charge injected into the polymer, and the resulting current is defined as space charge-limited current (SCLC). Space charges in materials may arise from several sources, such as (1) electrode injection of electrons and/or holes, (2) ionized dopants in interfacial depletion regions and (3) accumulation of mobile ions at electrode interfaces. Traps may be present in bulk materials or at interfaces and can result in lower carrier mobility. When present at interfaces, traps can also affect charge injection into materials. The electrical switching behaviors of some polymeric materials have been reported to be associated with space charges and traps. $^{44}$

\section{Filament conduction}

When the ON state current is highly localized to a small area within a memory device, the phenomenon can be termed 'filament conduction'. It has been suggested that filament conduction is confined to resistive random access memory devices in which the material undergoes physical damage. Two types of filament conduction have been widely reported in polymer resistive memory devices, and the formed filaments could be observed under an optical microscope or scanning electron microscope. ${ }^{55,56}$ One type consists of carbon-rich filaments formed by the local degradation of polymer films. ${ }^{56,57}$ The other is associated with metallic filaments that result from the migration of electrodes through polymer films. ${ }^{58,59}$ The production of metal filaments for filamentary conduction requires the use of polymers with both coordinating atoms and $\pi$-conjugation that can bind to metal ions regardless of the binding sites (side chain or main chain). ${ }^{33,60}$ Therefore, the filamentary conduction mechanism has often been suggested to explain switching phenomena in a variety of polymer memory devices.

\section{HPPS FOR VOLATILE MEMORY DEVICES}

For volatile memory effects, the device cannot be maintained in the ON state and will relax to the OFF state after the power is turned off. Nevertheless, the ON state can be maintained by refreshing the voltage pulse. Volatile memory effects can be divided into DRAM and SRAM, depending on the retention time of the ON state after removal of the applied voltage. For DRAM behavior (Figure 1), ${ }^{53}$ the ON state can

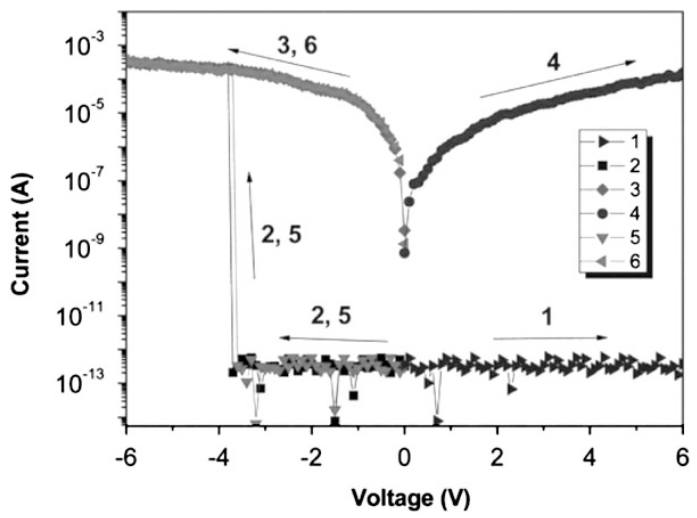

Figure 1 Current-voltage $(I-V$ characteristics of the ITO/polymer/AI memory device demonstrating DRAM behavior. The fifth sweep was conducted approximately $15 \mathrm{~s}$ after turning off the power. 
only be retained for a short time (less than $1 \mathrm{~min}$ ) after the removal of the applied voltage. For SRAM behavior (Figure 2), ${ }^{53}$ the device can stay in the ON state for a longer period of time after turning off the power than in DRAM devices. Despite the longer retention time of the ON state in SRAM devices, it is still volatile, and the ON state can relax to the OFF state without an erasing process.

\section{DRAM properties}

Based on the CT mechanism, Kang and colleagues ${ }^{43}$ first reported DRAM characteristics for the TPA-functionalized PI P9 (Scheme 6) in an Al/P9/ITO device. The $I-V$ curve shows that the memory device can be switched from a low-conductivity (OFF) state to a highconductivity $(\mathrm{ON})$ state and remain in this high-conductivity state during the subsequent positive scan with an ON/OFF current ratio of up to $10^{5}$. The ON state can be switched back to the OFF state during the subsequent negative sweep, revealing erasable memory behavior. After the power was turned off for $1 \mathrm{~min}$, the $\mathrm{ON}$ state relaxed to the steady OFF state without an erasing process, revealing volatile DRAM behavior; the device could subsequently be reprogrammed to the $\mathrm{ON}$ state. Moreover, the ON state could be electrically sustained by applying a refreshing voltage pulse of $1 \mathrm{~V}$ every $5 \mathrm{~s}$. According to theoretical calculations, the formation of unstable CT states is responsible for the observed DRAM behavior. Kang and colleagues ${ }^{61}$ described the PI P10, which has TPA-substituted diphenylpyridine

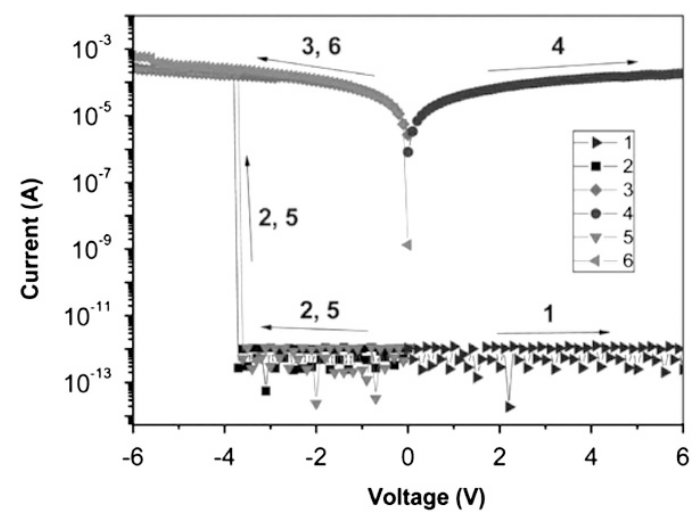

Figure 2 Current-voltage (I-V) characteristics of the ITO/polymer/AI memory device demonstrating SRAM behavior. The fifth sweep was conducted approximately $3 \mathrm{~min}$ after turning off the power. moieties and exhibits DRAM behavior with lower threshold voltages and a more volatile (less stable) ON state than P9. P11, which contains the electron acceptor 6FDA and electron donor carbazole, was synthesized by Qi and colleagues, ${ }^{62}$ and the electrical switching results of the sandwiched PI memory device ITO/P11/Au indicate that P11 possesses electrical bistability with two accessible conductivity states. The memory device can be reversibly switched from the OFF state to the ON state with an ON/OFF current ratio of approximately $10^{4}$, and the ON state of the device was lost immediately after removing the applied voltage; however, the ON state could be electrically sustained by applying a constant bias $(\sim 3 \mathrm{~V})$. The roles of the $\mathrm{D}$ and $\mathrm{A}$ components in the PI main chain were elucidated through molecular simulation.

\section{SRAM properties}

Oxadiazole-based polymers are typical electron-transporting materials in light-emitting devices. However, when bonded to stronger electron acceptors, such as itaconimide or maleimide, the oxadiazole groups can also act as electron donors. Thus, a solution-processable functional PI P12 (Scheme 7), containing oxadiazole and phthalimide moieties, was synthesized and reported by Kang and colleagues. ${ }^{63}$ During electrical sweeps, memory devices based on the ITO/P12/Al sandwich structure exhibit volatile bistable electrical switching SRAM behavior. As elucidated from the DFT simulation, the oxadiazole unit can act as an electron donor in the presence of a phthalimide moiety with a higher electron affinity. Electric field-induced CT from the oxadiazole donor to the phthalimide acceptor gives rise to a conductive CT state, which accounts for the transition of the device from the OFF state to the ON state. The conformational change induced by the electric field gives rise to a potential barrier to the back transfer of electrons, leading to a temporary retention of the $\mathrm{ON}$ state. As the small dipole moment of P12 in the ON state probably cannot maintain the conformationcoupled CT state, the ON state is volatile and is eventually lost after the applied voltage is removed. The conformation-coupled CT mechanism was further elucidated using molecular simulations of P12 in the ground and excited states and changes in the absorption spectra of P12 film in the ON and OFF states.

Zhang and Xu's group produced a series of PIs (P13a-d) by polymerizing diamine with a rigid nonplanar triphenylethylene conjugated moiety and four dianhydrides. ${ }^{64,65}$ These PIs exhibited interesting fluorescent and resistive switching (ON-OFF) characteristics, low dielectric constants, exceptional thermal stabilities and excellent mechanical properties, mainly due to their rigid non-

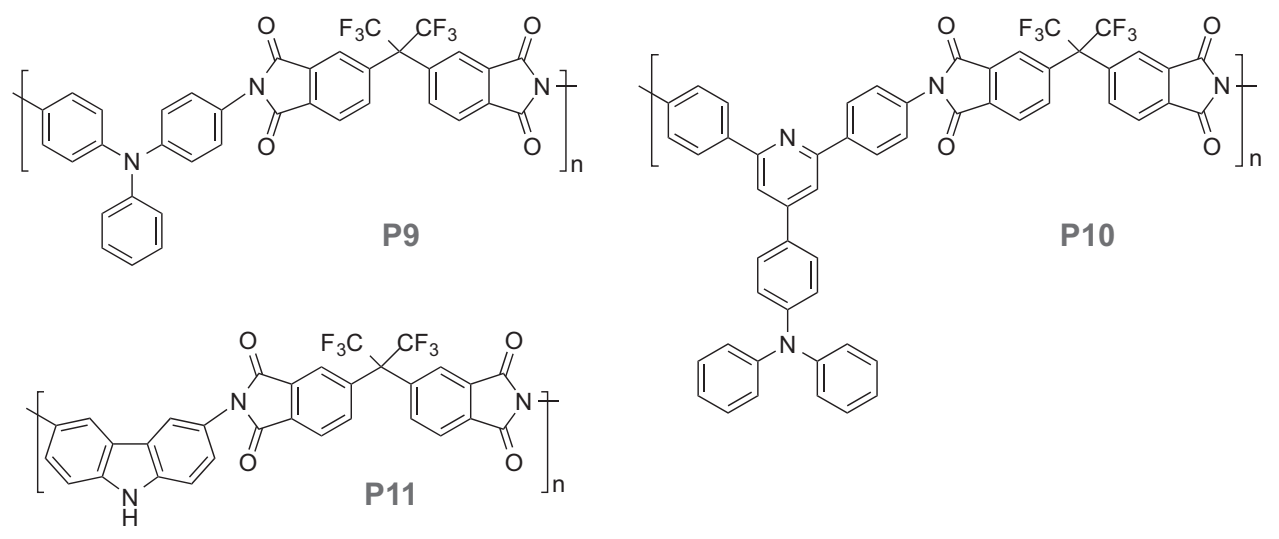

Scheme 6 Chemical structures of some polymers with DRAM memory properties. A full color version of this scheme is available at Polymer Journal online. 

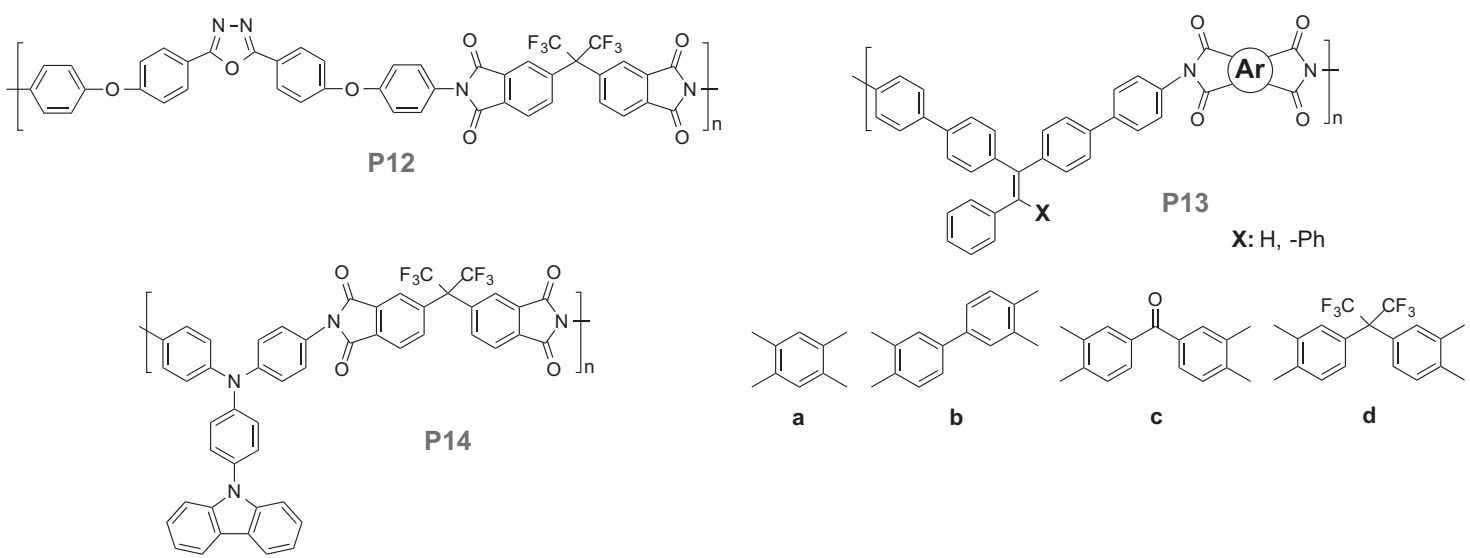

Scheme 7 Chemical structures of some polymers with SRAM memory properties. A full color version of this scheme is available at Polymer Journal online.

planar-conjugated structures. Memory devices with the ITO/P13/Al configuration exhibited the distinct volatile memory characteristics of SRAM. Theoretical analysis showed that the chemical and steric structures of the dianhydride might affect the electron distribution in the molecular orbital, and the CT mechanism could be used to explain the memory characteristics of the aromatic rigid nonplanar conjugated structure and thereby to build high-performance functional PIs.

The functional PI P14, which has a carbazole-tethered TPA unit as the electron-donating moiety and 6FDA as the electron-accepting species, was first synthesized by Liou et al. ${ }^{66}$ An ITO/P14/Au memory device fabricated by Qi's group ${ }^{67}$ exhibited two bi-directionally accessible conductivity states during both the positive and negative voltage sweeping processes, and achieved an ON/OFF current ratio as high as approximately $10^{5}$ with no polarity except for a trivial variation in the switching voltage. Moreover, both the ON and OFF states were stable under a constant voltage stress of $-1 \mathrm{~V}$ and survived for up to $10^{8}$ read cycles with a periodic read pulse of $-1 \mathrm{~V}$. The $I-V$ characteristics of the device in the OFF state agree fairly well with the SCLC model, while the characteristics in the ON state also agree well with the ohmic conduction model. The roles of the D and A units in the memory mechanism of the electroactive PI were elucidated via molecular simulation.

\section{HPPS FOR NON-VOLATILE MEMORY DEVICES}

Non-volatile memory devices can stay in the ON state steadily without an applied voltage bias. Non-volatile memory behaviors can be divided into two classes, namely WORM memory and rewritable (flash) memory, depending on whether a suitable voltage can switch the ON state to the OFF state. If the ON state can be switched back to the OFF state by applying a suitable voltage, which is an erasing process, then the memory effect is called rewritable memory (Figure 3). ${ }^{68}$ However, WORM memory is capable of maintaining the ON state (holding data) permanently, even after the application of a reverse voltage, and can be read repeatedly (Figure 4$).{ }^{53}$

\section{WORM properties}

Ree and colleagues reported a PI P15 (Scheme 8) with a pendant hydroxyl group on each TPA group. ${ }^{69}$ An ITO/P15/Al device exhibited WORM memory behavior with a high ON/OFF current ratio of up to $10^{6}$, a long retention time and a low threshold voltage. The WORM characteristics were found to persist even at high temperatures of up to $150^{\circ} \mathrm{C}$, demonstrating the excellent thermal stability of PI-based

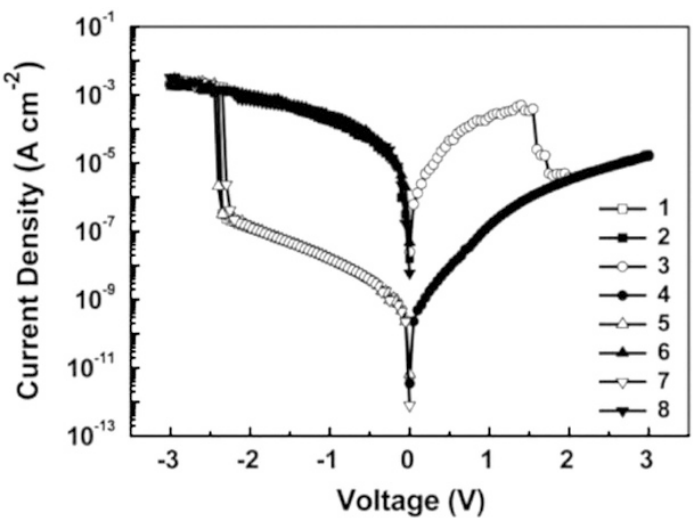

Figure 3 Current-voltage $(I-V$ characteristics of the ITO/polymer/AI memory device demonstrating flash behavior.

memory devices. For the memory mechanism, the fitted $I-V$ data showed that a trap-limited SCLC mechanism was dominant when the device was in the OFF state. Moreover, the ON state current level of the device was found to be independent of the device cell size, suggesting that the electrical transition in the device is due to filament formation inside the active polymer layer. After the filaments have formed, they act as channels through which the carriers flow by means of a hopping process, leading to the ON state.

The thermally stable PI P16, which has a pendant spiropyran moiety, was designed as a functional material and fabricated into a memory device; its optoelectrical dual-mode memory was studied by Song and colleagues. ${ }^{70}$ In an $\mathrm{Al} / \mathrm{P} 16 / \mathrm{Al}$ device, the memory exhibits WORM behavior with an ON/OFF current ratio of approximately $10^{4}$. These properties open up the possibility of potential application of multimode data storage memory devices with thermally stable PIs as the functional medium.

Wang et al. ${ }^{71}$ synthesized a series of functional PIs P17a-e with different phthalimide electron acceptors, which showed excellent thermal stability and high glass transition temperatures $\left(T_{\mathrm{g}}\right)$. Memory devices based on P17a thin films exhibited symmetric bistable WORM memory behaviors with switching threshold voltages of $\pm 1.8 \mathrm{~V}$. The $\mathrm{ON}$ states of the devices are nonvolatile and can withstand a constant voltage stress of $-1 \mathrm{~V}$ for $6 \mathrm{~h}$ and $10^{8}$ pulse read cycles under ambient conditions. Such electrical switching behavior can be attributed to electric field-induced electron transfer from the TPA donor to the 


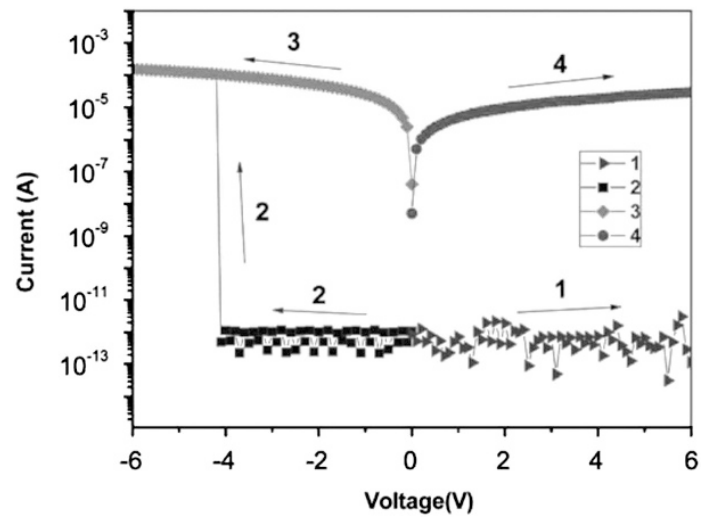

Figure 4 Current-voltage ( $I-V$ characteristics of the ITO/polymer/AI memory device demonstrating WORM behavior.

phthalimide acceptor through the oxadiazole spacer. The excellent memory performance further demonstrates the advantages of PI derivatives for memory device applications. A series of PIs P18a-f containing TPA-substituted triazole moieties were developed, ${ }^{72}$ and a memory device based on polymer P18a exhibited WORM memory behavior with excellent device performance, such as a high ON/OFF current ratio $\left(10^{5}\right)$ and good stability. According to theoretical calculations, the memory mechanism can be assigned to electric field-induced 'conformation-coupled CT'. The TPA moieties act as the electron donors and the phthalimide moieties act as the electron acceptors, which are linked by a single bond. Thus, the conformationcoupled CT process can probably occur under an electric field. The twisted molecular chain in the CT state can give rise to a large potential energy barrier for the dissociation of CT complexes. As a result, the conductive CT state is stabilized, and the high conductivity $(\mathrm{ON})$ state is retained. Once a high-conductivity state is formed, carriers can move in both directions. Thus, a reverse bias of the same magnitude will not return the device to the OFF state.

Another series of PIs P19a-d containing carbazole as the electron donor and different phthalimides as the electron acceptors were synthesized by Ree and colleagues. ${ }^{73}$ All of the polymers exhibited high thermal and dimensional stability. In particular, P19a and P19b revealed excellent chemical resistance to organic solvents, which is very promising for the fabrication of high-performance memory devices in three-dimensional, multi-stacked structures. These PI thin films can be fabricated by solution spin-coating of soluble poly(amic acid) precursors and subsequent thermal imidization and the PI devices exhibit excellent unipolar WORM memory behavior with an ON/OFF current ratio as high as $10^{9}-10^{10}$ in both positive and negative voltage sweeps with turn-on threshold voltages at $2.3-3.3 \mathrm{~V}$, depending on the chemical structures of the polymers. According to the fitted $I-V$ data, the trap-limited SCLC mechanism is dominant in the OFF states of the devices at high voltages, and ohmic conduction is dominant both in the OFF state at lower voltages and in the ON state.

Qi and colleagues described the aromatic hyperbranched PI P20 in which TPA moieties function as electron-donating groups and 6FDA serves as electron-accepting species. ${ }^{74}$ A fabricated memory device with a simple ITO/P20/Au sandwich structure exhibited two distinct but irreversible conductivity states with a threshold transition voltage at approximately $2.0 \mathrm{~V}$ and ON/OFF current ratio of approximately 300. The non-volatile and irreversible electrical WORM memory effect observed was ascribed to electric field-induced CT interactions between TPA and 6FDA groups and the strong electron affinity of
6FDA units, which supposedly possess the ability to retain the chargeseparation state of the generated CT complexes.

\section{Flash properties}

By changing the hole-transporting and electron-donating moieties, four PI derivatives containing diphenylcarbamyloxy (P21) ${ }^{75}$ diphenylaminobenzylidenylimine $(\mathbf{P} 22)^{76}$ and carbazole $\left(\mathbf{P} 23^{77}\right.$ and $\left.\mathbf{P} 24^{78}\right)$ on their side chains have also been synthesized for use as excellent rewritable memory devices (Scheme 9). For example, P21 exhibits memory performance with a high ON/OFF current ratio of up to $10^{9}$, low power consumption and long retention time in both the $\mathrm{ON}$ and OFF states. ${ }^{75}$ The observed rewritable memory behavior for $\mathbf{P} 22$ is due to Schottky emission and local filament formation. ${ }^{76}$ For polymer P23, the filamentary mechanism has been demonstrated by in situ conductive atomic force microscopy measurements. ${ }^{77}$ A memory device based on P24 exhibits excellent unipolar ON and OFF switching behavior with very low power consumption and a high ON/OFF current ratio of up to $10^{11} .{ }^{78}$

According to Ueda and Chen's study, ${ }^{34}$ hole-transporting and electron-donating moieties, such as sulfur-containing, TPA and phenyl groups, could also be introduced into the main PI chain. Electron-rich sulfur-containing groups generally have high dipole moments as well as good electron-donating and charge-transporting characteristics. Two sulfur-containing PIs P25 and P26 with APTT (2,7-bis(phenylenesulfanyl)thianthrene) and SDA (4,4'-thiobis( $p$-phenylenesulfanyl)) moieties, respectively, in their main polymer chains have been synthesized for memory device applications. ITO/PIs/Al memory devices containing these PIs showed non-volatile flash memory characteristics with low turn-on threshold voltages of $1.5 \mathrm{~V}$ (P25) and $2.5 \mathrm{~V}$ (P26). The ON/OFF current ratios of the devices were approximately $10^{4}$ in ambient atmosphere. According to theoretical analysis, a field-induced CT mechanism between the APTT/3SDA and 6FDA moieties could be used to explain the memory characteristics of these polymers. The high dipole moments of P25 and P26 result in stable CT complexes even after the driving power is turned off; thus, the ON state of these devices could remain. However, a reverse bias applied to P25 or P26 could dissociate the CT complex and return it to the OFF state, leading to the flash memory behaviors.

Ree and colleagues ${ }^{79}$ generated PI P27 by introducing anthracene as the hole-transporting and electron-donating moiety on the side chain. This polymer, which is organo-soluble and has excellent thermal and dimensional stability, could be easily fabricated into high quality thin films by spin coating and subsequent drying. An Al/P27/Al memory device exhibited excellent non-volatile and rewritable bipolar and unipolar switching behaviors over a very small voltage range of less than $\pm 2 \mathrm{~V}$ with a high ON/OFF ratio of up to $10^{7}$. The device could be written, read and erased repeatedly with long-term reliability in ambient air conditions as well as at high temperatures of up to $200^{\circ} \mathrm{C}$. Additionally, this device can be produced in three-dimensional arrays for very high density storage. By fitting the $I-V$ data for the OFF and ON states, the memory mechanism of the device was assigned to traplimited SCLC and local filament formation. Furthermore, a polymer similar to P27 without anthracene exhibited no electrically bistable characteristics. Hence, both the anthracene moieties, which act as hole trapping sites, and the phthalimide moieties, which act as electron trapping sites, in polymer $\mathbf{P} 27$ have key roles in the electrical switching properties. In addition, a device with an $\mathrm{Au}$ bottom electrode ( $\mathrm{Au} / \mathrm{PI} /$ $\mathrm{Al}$ device) was found to exhibit a switching behavior similar to that of the device with an $\mathrm{Al}$ bottom electrode.

In addition, Li et al. ${ }^{80}$ designed and synthesized two aromatic PIs P28 and P29 with pendant carbazole and TPA-substituted groups, 

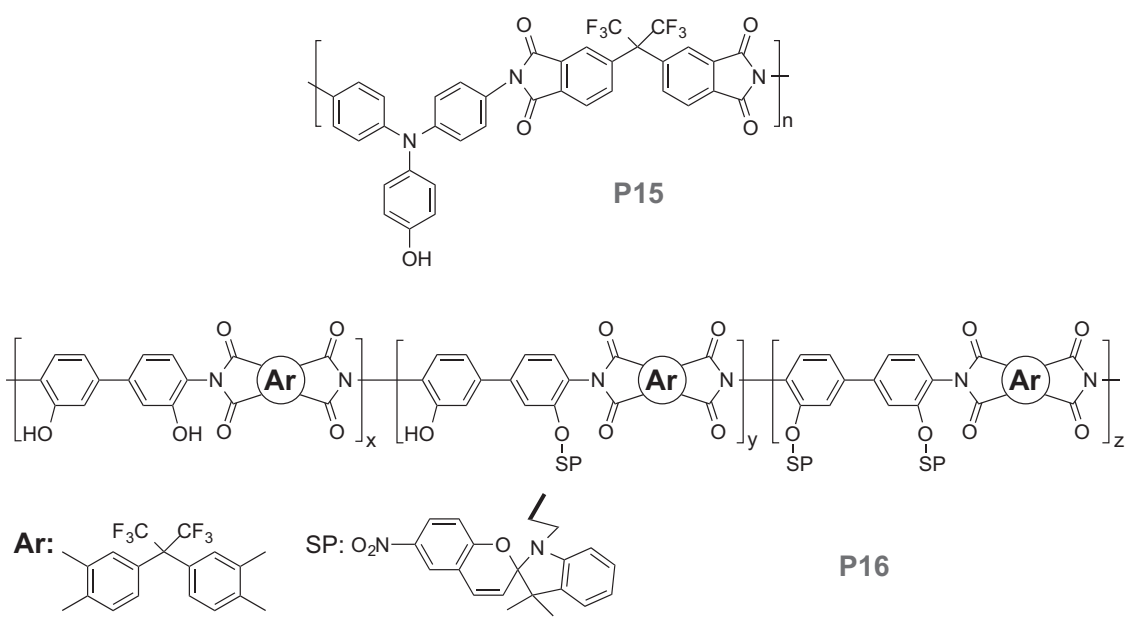

P16<smiles>CN(C)C(=O)C1CC2(C)C3C=CC(C(=O)N(C)C3=O)C2C(=O)N1c1cc(-c2nnc(-c3ccc(N(c4ccccc4)c4ccccc4)cc3)o2)cc(C(C)(C)C)c1</smiles>

Ar:<smiles>CCc1cc(C(F)(F)C(F)(F)F)ccc1CCc1ccc(C)c(F)c1</smiles>

a<smiles>Cc1ccc(C(=O)c2ccc(C)c(C)c2)cc1C</smiles><smiles>CCn1c(=O)c2cc3c(=O)n(-c4ccc(-c5ccc(C(C)(C)C)c(OCCn6c7ccccc7c7ccccc76)c5)cc4OCCn4c5ccccc5c5ccccc54)c(=O)c3cc21</smiles>

Ar:<smiles>Cc1cc(C)c(C)cc1C</smiles><smiles>Cc1ccc(OCCOc2ccccc2)cc1C</smiles>

c<smiles>Cc1ccc(S(=O)(=O)c2ccc(C)c(C)c2)cc1C</smiles>

d

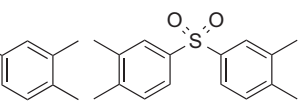

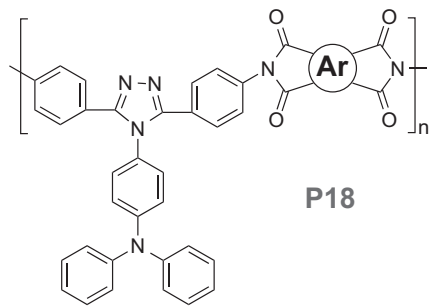

Ar: $\quad \mathrm{F}_{3} \mathrm{C} \quad \mathrm{CF}_{3}$<smiles>Cc1ccc(S(=O)(=O)c2ccc(C)c(C)c2)cc1C</smiles>

a

b<smiles>Cc1ccc(-c2ccc(C)c(C)c2)cc1C</smiles><smiles>Cc1cc(C)c(C)c(C)c1C</smiles>

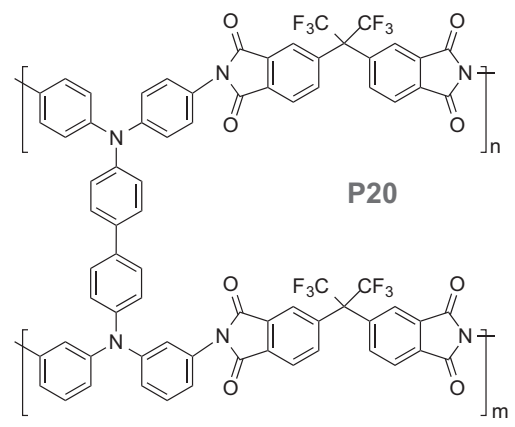

Scheme 8 Chemical structures of some polymers with WORM memory properties. A full color version of this scheme is available at Polymer Journal online.

respectively, as donors for the fabrication of memory devices. Both PIs showed good solubilities, fine film-forming capabilities and excellent thermal stabilities. A device based on a P28 film exhibited bipolar rewritable flash memory characteristics with a turn-on voltage at around $+2.7 \mathrm{~V}$ and a turn-off voltage at $-1.5 \mathrm{~V}$. On the other hand, a device based on a P29 film demonstrated unipolar rewritable flash memory behavior with a turn-on voltage at around $\pm 2.2 \mathrm{~V}$ and a turn-off voltage around $\pm 1.0 \mathrm{~V}$. The ON/OFF current ratios of the devices were approximately $10^{4}$. The current of the OFF state in the $\mathrm{Al} / \mathbf{P 2 8} / \mathrm{ITO}$ device was fitted using the SCLC model, whereas that of the Al/P29/ITO device was fitted to the Schottky emission model. Both of the ON-state currents were well fitted by the ohmic model.

Ferrocene is also a typical electron donor. P30 grafted with ferrocene, in which phthalimide can act as an electron acceptor, was synthesized by $\mathrm{Wu}$ and colleagues. ${ }^{81}$ The resulting P30 exhibits electrical bistability and non-volatile memory behavior with an 

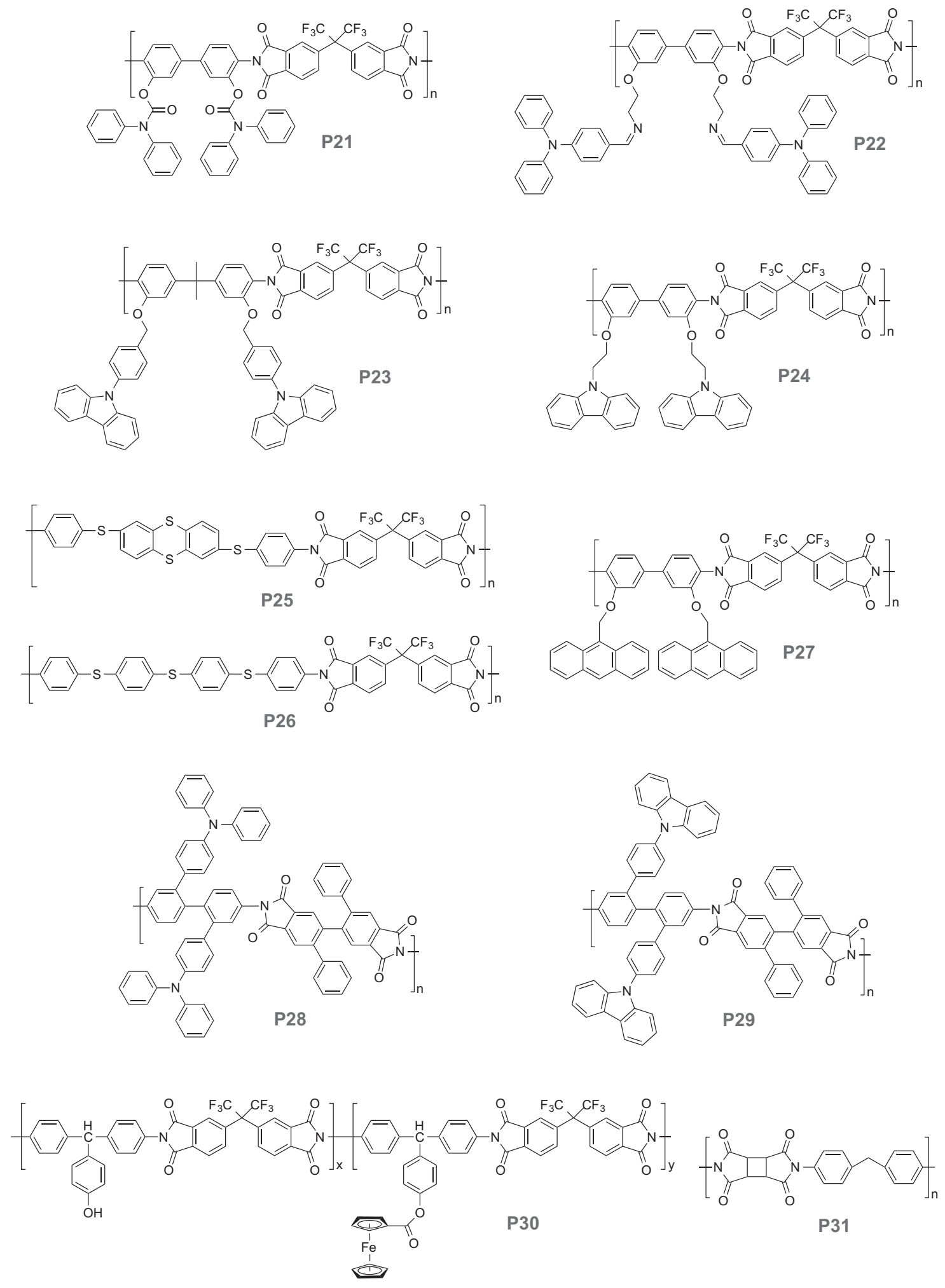

Scheme 9 Chemical structures of some polymers with flash memory properties. A full color version of this scheme is available at Polymer Journal online.

ON/OFF current ratio of approximately $10^{3}$. The CT mechanism was reasonably interpreted using the thermionic emission and SCLC models.

Most recently, Tsai and colleagues ${ }^{82}$ described a new P31-containing D-A system, which exhibited non-volatile memory behavior. P31 exhibits asymmetric bipolar switching with a high ON/OFF current ratio of $>10^{5}$. The switching mechanism of the P31 layer could be attributed to the formation and dissociation of a CT complex. When a positive voltage is applied, the electron-donor and electron-acceptor undergo an electron transfer interaction to form a conductive CT 
complex. By contrast, when the opposite voltage is applied, the conductive CT complex is dissociated, and the device returns to its initial low conductivity state. Furthermore, the switching properties of P31 are related to the thickness of the P31 layer, and the ON/OFF current ratio increases with increasing thickness. However, the active area also affects the stability of the device. That is, a small P31 device is more stable than a large device. In addition, the molecular chain lengths of PI dominate the electron conduction capability in the low and high conductivity states, affecting the switching properties. Therefore, molecular chain lengths are an important issue in PI memory research.

\section{EFFECTS OF THE MOLECULAR DESIGN ON VOLATILITY}

For resistive memory devices, the writing operation is performed by applying a voltage bias or pulse to the device, resulting in switching between the high-resistance (OFF) and low-resistance (ON) states. Memory devices show non-volatile or volatile memory behavior depending on whether electrical power is required to maintain the given state (ON state). In addition, memory properties can be tuned by changing the following properties: the electron donor or acceptor in functional donor-acceptor type polymers, linkage, conformation and film thickness of the active polymer layer within the devices. ${ }^{48,83-85}$

\section{Donor effect}

Shen and colleagues synthesized polymers P32/P33 (Scheme 10) consisting of alternating electron-donating carbazole/TPA moieties and electron-accepting phthalimide moieties. ${ }^{45}$ These polymers are thermally stable with $5 \%$ weight loss over $500^{\circ} \mathrm{C}$ and $T_{\mathrm{g}}$ above $290^{\circ} \mathrm{C}$. A memory device with the configuration Al/carbazole-based P32/ITO showed flash memory behavior with low turn-on and turn-off threshold voltages of -1.6 and $+2.2 \mathrm{~V}$, respectively, whereas a memory device composed of Al/TPA-based P33/ITO exhibited excellent WORM memory performance, such as a low turn-on threshold voltage around $\pm 2.7 \mathrm{~V}$. Both devices exhibited high ON/OFF current ratios of approximately $10^{6}$ and long retention times of $10^{4} \mathrm{~s}$ even in ambient atmosphere. According to the DFT calculations, the switching mechanisms of CT between the electron donor and acceptor moieties and the electric field-induced conformational ordering of the polymer side chains/backbones were responsible for the memory behaviors.

According to Shen and Ding's research, PI P34 ${ }^{86}$ containing $3^{\prime}, 4^{\prime}, 5^{\prime}$-trifluorobiphenyl and phenyl moieties as the electron donors was synthesized, and the fabricated Al/P34/ITO devices exhibited a WORM-type memory behavior with a low turn-on threshold voltage of $-1.7 \mathrm{~V}$ and an ON/OFF current ratio of $10^{4}$. The $I-V$ curves in the $\mathrm{OFF}$ and $\mathrm{ON}$ state were fitted according to various theoretical models. In addition, the charge transport in the OFF state was governed by the Poole-Frenkel model and that in the ON state was governed by the ohmic model. Similar to polymer P34, methoxy-containing PI P35, ${ }^{87}$ which has excellent solubility and high thermal and dimensional stabilities, was also synthesized for the investigation of memory behavior. A memory device with the configuration Al/P35/ITO exhibited bipolar WORM memory behavior with a low switching-on voltage $(<3 \mathrm{~V})$. According to the theoretical calculations, the relatively strong dipole moments of the methoxy-containing PI P35 produced a more stable CT complex as a WORM memory device compared with P34.

Shen and Ding also prepared another PI P36 ${ }^{86}$ by introducing $3^{\prime}, 4^{\prime}, 5^{\prime}$-trifluorobiphenyl and phenyl moieties as the electron donors. The fabricated Al/P36/ITO device has a flash type memory behavior with 'write' and 'erase' voltages of about -1.3 and $4.0 \mathrm{~V}$, respectively. A series of PIs P37a-c ${ }^{87}$ with the same main chain but different electron-donating groups at the 2,2'-position of the biphenyl moieties also exhibited bipolar flash-type memory characteristics in Al/P37/ITO devices with low switching-on and switching-off voltages $(<3 \mathrm{~V})$. According to theoretical calculations, the CT mechanism could be used to explain the memory characteristics of these PIs.

Two thermally and dimensionally stable aromatic TPA-based PIs bearing electron-accepting bis(trifluoromethyl)phenyl groups (P38) and electron-donating bithiophene groups (P39) were synthesized (Scheme 11). ${ }^{88}$ In the Al/PI/Al devices, all of the PIs initially exhibited a high resistance (OFF state). Under positive and negative voltage sweeps, the PIs demonstrated various memory behaviors (volatile DRAM or nonvolatile WORM memory) depending on the substituents of the TPA units. The electron-donating bithiophene substituents increased the nucleophilicity and aromatic resonance power (that is, $p$ conjugation length) of the TPA unit, improving the stability of the memory behavior. In contrast, the bis(trifluoromethyl)phenyl substituents significantly reduced the nucleophilic power of the TPA units, reducing the stability of the memory behavior. All of the PI films revealed high ON/OFF current ratios $\left(10^{5}-10^{8}\right)$ and high stabilities in both the OFF and ON states, even under ambient air conditions. The memory behaviors were governed by a mechanism involving fieldenhanced thermal emission of trapped charges and filament formation.

Three functional PIs, P40, P41 and P42, bearing conjugated bis (triphenylamine) (2TPA) derivatives with electron-donating and accepting substituents were synthesized. ${ }^{89} \mathrm{Al} / \mathrm{PI} / \mathrm{Al}$ devices containing these PIs initially had high resistances (OFF state). However, under positive and negative voltage sweeps, the PIs manifested volatile or non-volatile digital memory behaviors depending on the substituents on the 2TPA unit. The 2TPA-based PI P41 bearing 2TPA with electron-donating methoxy substituents showed unipolar WORM memory behavior, whereas PI P42 containing electron-accepting cyano groups exhibited unipolar DRAM behavior with high ON/OFF current ratios $\left(10^{6}-10^{10}\right)$ even under ambient air conditions. The memory behaviors were found to be governed by a mechanism involving trap-limited space-charge limited conduction and local filament formation by taking into consideration the chemical nature and molecular orbital levels of the PIs, possible trapping sites, substituent effect and work functions of the metal electrodes. Overall, this study demonstrated that these thermally stable PIs are highly suitable for the low-cost mass production of high-performance digital memory devices that can be operated with very low power consumption. Moreover, the memory mode can be tuned by changing the substituent on the 2TPA unit.

To further investigate the effects of modified TPA-containing PIs on the performances of memory devices, a series of DSDA $\left(3,3^{\prime}, 4,4^{\prime}\right.$ diphenylsulfonyltetracarboximide)-based PIs P43-P46 ${ }^{90}$ bearing three different TPA derivatives were synthesized and found to have high thermal and dimensional stabilities compared with conventional PIs. Al/PIs/Al devices containing P43-P46 initially exhibited high resistance, and polymers P43-P46 exhibited various memory behaviors under positive and negative voltage sweeps depending on the substituents on the TPA units. Interestingly, for P44, only $\sim 80 \%$ of the device cells showed unipolar WORM memory behavior, and the other device cells showed unipolar DRAM behavior, which can be attributed to the cooperative role played by the cyano substituents as charging sites and electron-accepting groups. However, polymer P46 showed rewritable memory behavior. All of the PIs switched on at approximately $\pm 2 \mathrm{~V}$. They all showed excellent retention abilities in 

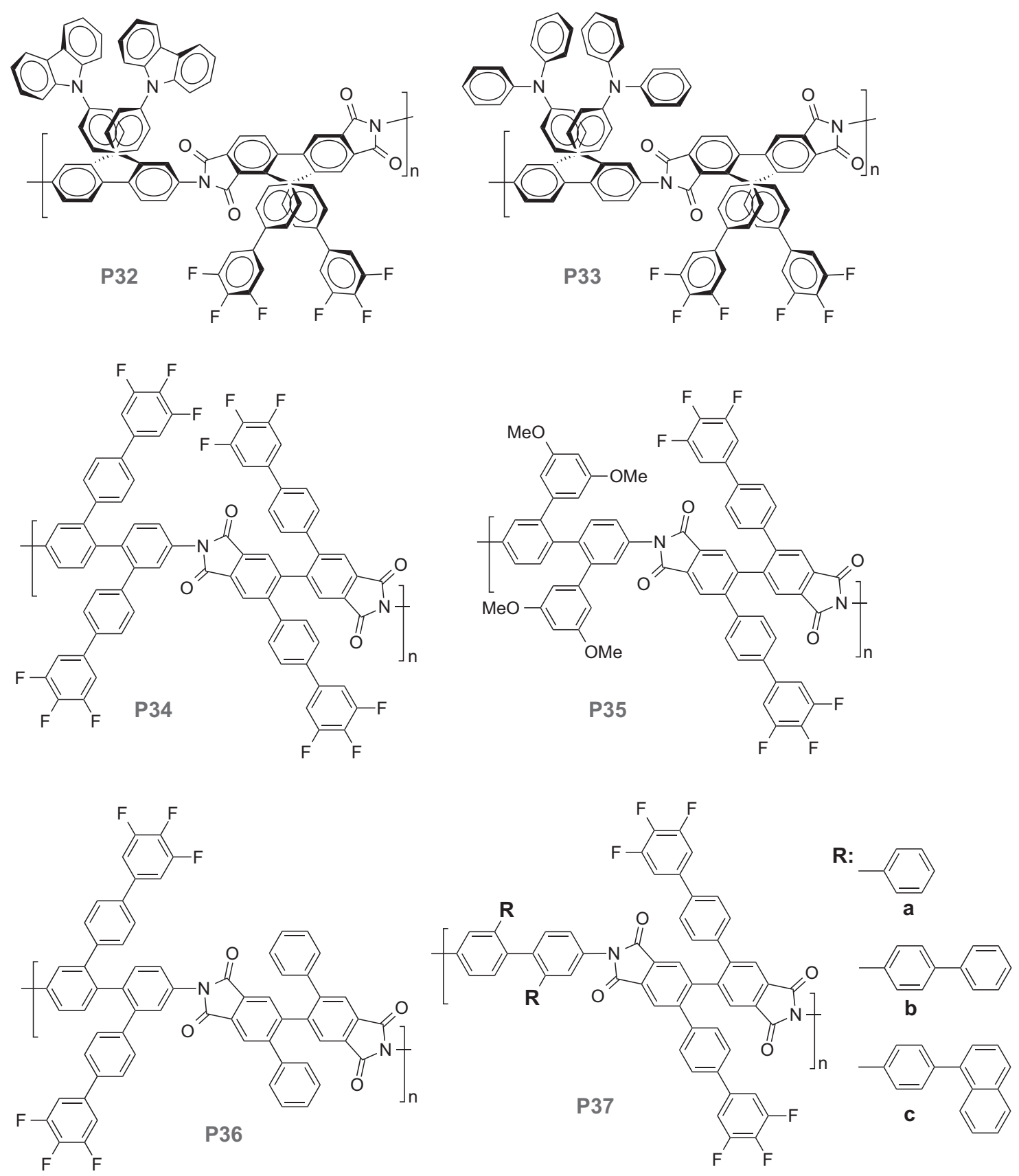

Scheme 10 Chemical structures of some polymers with donor effects. A full color version of this scheme is available at Polymer Journal online.

both the OFF and ON states, even under ambient air conditions, and high ON/OFF current ratios $\left(10^{6}-10^{8}\right)$. The memory behaviors were found to be governed by mechanisms involving trap-limited SCLC conduction and local filament formation.

Our group ${ }^{85}$ further synthesized two PIs (P47 and P48) by modifying the TPA with different substituents to optimize the polymer structures. The polymers displayed different non-volatile memory behaviors in $\mathrm{Al} / \mathrm{PI} / \mathrm{Al}$ devices depending on the substituents of the TPA unit. Polymer P47 showed unipolar WORM memory behavior, whereas polymer P48 film showed unipolar and bipolar switching memory behavior. Both polymers showed excellent stability in both the OFF and ON states, even under ambient condition, with ON/OFF current ratios of up to $10^{8}-10^{9}$. The memory behaviors of these two polymers can be attributed to trap-limited space-charge limited conduction and local filament formation. The unipolar WORM memory characteristics of polymer P47 can be attributed to the enhanced electron-donating ability of the TPA unit resulting from three methyl substituents, which acted as effective charge-trapping sites. In comparison, the unipolar and bipolar switching memory characteristics of polymer $\mathbf{P 4 8}$ originated from the dimethylamine substituents, which act as charge-trapping sites and enhance the electron-donating ability of the TPA unit. These results demonstrated that the memory modes of PIs can be tuned by changing the substituents on the TPA unit. 

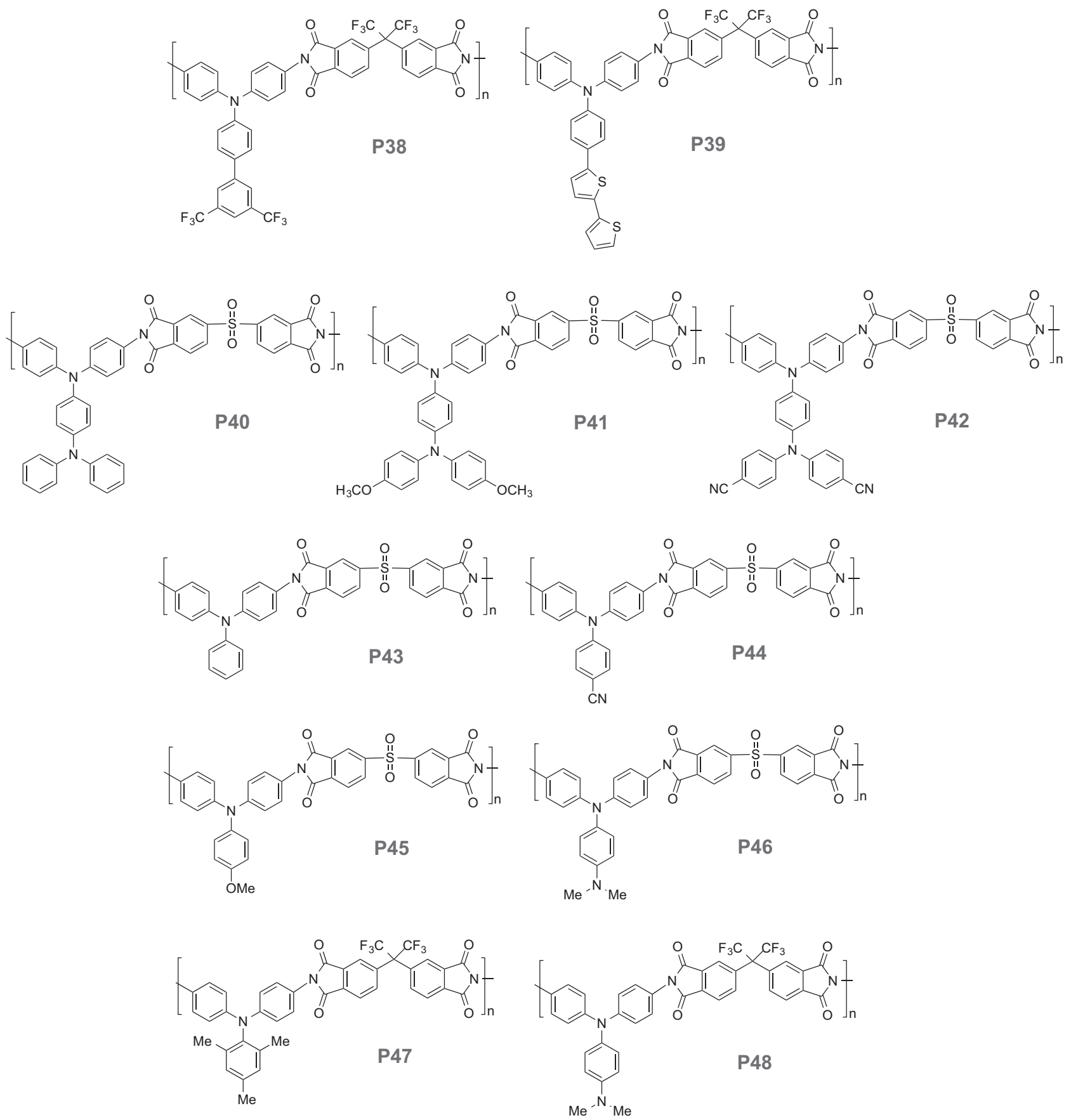

Scheme 11 Chemical structures of some polymers with donor effects. A full color version of this scheme is available at Polymer Journal online.

Subsequently, our group designed and prepared another aromatic PI $\mathbf{P}^{53}$ by introducing highly electron-donating starburst triarylamine groups. The incorporation of starburst triarylamine groups on the polymer main chain not only stabilizes its radical cations but also enhances the solubility and film-forming capability of the resulting PIs. In addition, the obtained PI possessed high $T_{\mathrm{g}}$ and good thermal stabilities, which have important roles in resistive memory devices. To investigate the effects of the donor moieties in PIs on their memory behaviors, the corresponding P6 (3Ph-PIs) and P5 (5Ph-PIs) were also prepared and studied. As the intensity of the electron donation increases from P6 to P5 and then to P7, the retention time of the memory device reveals a systematic increase.

\section{Acceptor effect}

A new series of aromatic PIs P49a-P49c ${ }^{91}$ was synthesized via two-step polymerization from newly synthesized tetracarboxylic dianhydride monomers reacting with $2,2^{\prime}$-bis $\left[4^{\prime}-\left(3^{\prime \prime}, 4^{\prime \prime}, 5^{\prime \prime}\right.\right.$-trifluorophenyl)phenyl]-4,4'-biphenyl diamine (Scheme 12). The resulting polymers exhibited excellent organo-solubility and thermal properties. In this study, each repeat unit of the polymers contained different aryl 
<smiles></smiles>

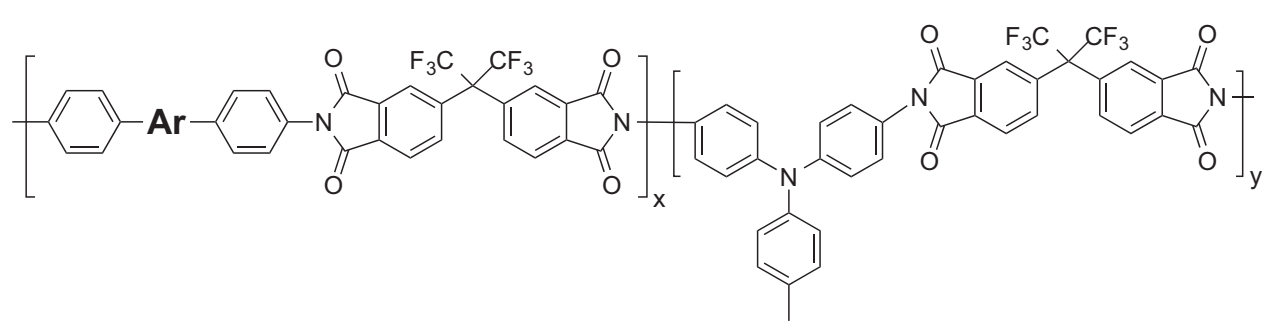

Ar:<smiles>CN1C(=O)c2ccc3c4ccc5c6c(ccc(c7ccc(c2c37)C1=O)c64)C(=O)N(C)C5=O</smiles>

P8<smiles>CN1C(=O)c2ccc3c4c(ccc(c24)C1=O)C(=O)N(C)C3=O</smiles>

P50<smiles>Cn1c(=O)c2cc3c(=O)n(C)c(=O)c3cc2c1=O</smiles>

P51

$x=1,2.5,5,10$

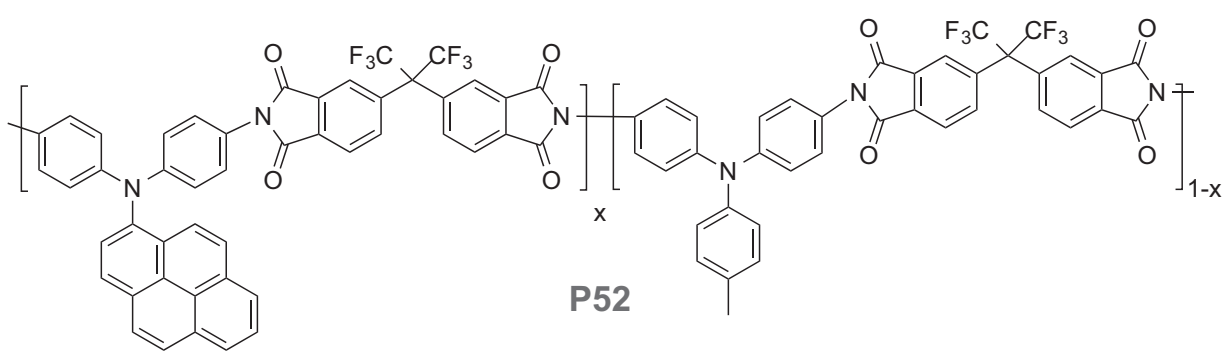

Scheme 12 Chemical structures of some polymers with acceptor effects. A full color version of this scheme is available at Polymer Journal online.

pendants in two imide rings with electric polarization characteristics. The aryl pendants are electron donors and nucleophilic sites, whereas imide rings are electron acceptors and electrophilic sites. Thus, all of these groups are likely to act as charge-trapping sites, depending on their associations. The fabricated sandwich structured Al/P49-a/ITO memory devices revealed flash-type memory behavior, whereas $\mathrm{Al} /$ P49-b/ITO and Al/P49-c/ITO devices exhibited WORM memory characteristics with different threshold voltages. In addition, Al/PIs/ ITO devices showed high stability under constant stress or a continuous read pulse voltage of $-1.0 \mathrm{~V}$. All of the devices have long retention times in ambient air conditions and ON/OFF current ratios of approximately $10^{3}$. This series of PIs exhibited a good combination of properties required for high-performance materials and demonstrated promising potential for polymeric memory applications.
Recently, our group reported a series of P5 $\mathrm{PIs}^{48}$ with different phthalimide moieties and found that differences in the HOMO and LUMO energy levels and dipole moments can significantly influence memory behaviors. PIs P5-DSPI and P5-NTPI exhibit non-volatile WORM memory behaviors in ITO/polymer/Al sandwiched devices with threshold voltages of -4.8 and $-4.9 \mathrm{~V}$ and high ON/OFF current ratios of $10^{9}$ and $10^{10}$, respectively. According to the theoretical analysis, the low LUMO energy level and the strong CT effect of P5-NTPI produce the WORM behavior. Interestingly, although P5-DSPI has a higher LUMO energy level, it also showed WORM behavior because of its high dipole moment of $5.45 \mathrm{D}$. By reducing the electron-withdrawing intensity of the phthalimide moieties, the retention time of the corresponding memory devices also decreased, as in P5-6FPI and P5-PMPI, which exhibit DRAM and SRAM 
behaviors, respectively. These results demonstrated that the energy level and polarity of PIs can be tuned by changing the electron acceptor, which can significantly influence memory performance.

Ueda and colleagues ${ }^{54,92}$ generated a series of random coPIs, P8, P50 and P51, by varying the feed ratio of acceptor units (PTI (perylenetetracarboxydiimide), naphthalenetetracarboxydiimide (NTI) and benzenetetracarboxydiimide (BTI), respectively). Memory devices prepared from these coPIs sandwiched between ITO and Al electrodes exhibited tunable electrical bistability from volatile to nonvolatile WORM memory characteristics with increasing perylene bisimide, NTI or BTI. Ueda and Chen summarized the results and studied the effects of the conjugation length of the acceptor moiety $(\mathrm{PTI}>\mathrm{NTI}>\mathrm{BTI})$ on the resulting memory characteristics. Stable CT complexes could be obtained through stabilization of the radical anion by the long conjugation and high electron affinity of the NTI and PTI moieties, leading to non-volatile WORM memory characteristics. However, owing to the low HOMO energy level of the BTI unit, a large energy barrier was generated that prevented hole injection from the $\mathrm{Al}$ electrode into the polymer, resulting in a lack of switching behavior at a high BTI composition. The study also showed that the conjugation lengths of the acceptor moieties significantly affected the memory characteristics of D-A coPIs.

New coPIs $\mathbf{P 5 2}{ }^{68}$ with main chain TPA/pyrene groups randomly copolymerized with 6FDA acceptors by chemical imidization were prepared by Ueda and colleagues and used as the active layer for flexible resistance memory devices in a cross-point $\mathrm{PEN} / \mathrm{Al} / \mathrm{P}$ 52/Al platform. The memory behaviors varied with the
$\mathrm{N}, \mathrm{N}$-bis(4-aminophenyl)aminopyrene molar content from volatile to non-volatile flash, flash/WORM and WORM memory for P52-0, P525, P52-10 and P52-100, respectively (where the numbers 0, 5, 10 and 100 are the molar percentages of the pyrene-based polyimide). Electrical bistability was ascribed to the CT as suggested by theoretical simulation, and the largely conjugated pyrene moiety gives excellent non-volatile electrical properties to the stable CT complex. The resistive switching characteristics have been successfully analyzed using a proper theoretical $J-V$ dependence model. The fabricated coPIs exhibited superior memory performance and a high level of mechanical durability when operated under various bending conditions. A possible switching mechanism based on the $\mathrm{CT}$ interaction was proposed through molecular simulation and fitted with physical conduction models in the OFF and ON states. Manipulation of the memory volatility by tailoring the molecular design and the obtained plastic electronic devices demonstrated promising applications of D-A PIs in integrated memory devices.

\section{Linkage effect}

Our group reported two TPA-based aromatic PIs (P53 and P54 $)^{93}$ containing pendent anthraquinone units that were directly attached to or incorporated via ether linkages into the backbones as electron acceptors, and compared the memory behaviors of these PIs (Scheme 13). Both of the PIs exhibited excellent thermal stabilities and high glass transition temperatures. ITO/P53/Al memory devices exhibited distinct volatile DRAM characteristics, whereas the ITO/P54/ Al device showed volatile SRAM properties. Thus, the results indicated<smiles></smiles>

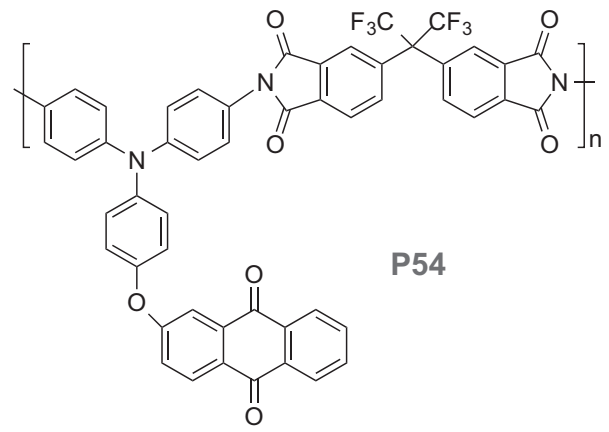<smiles>CC(C)(C)N1C(=O)c2ccc(C(c3ccc4c(c3)C(=O)N(c3ccccc3)C4=O)(c3ccc4c(c3)C(=O)N(c3ccccc3)C4(C)C)C(F)(F)F)cc21</smiles>

P55<smiles>CC(C)(c1ccc2c(c1)C(=O)N(c1ccc(Oc3ccc(N(c4ccccc4)c4ccccc4)cc3)c(C(F)(F)F)c1)C2=O)c1ccc2c(c1)C(=O)N(C(C)(C)C)C2=O</smiles><smiles>Cc1ccc(N(c2ccc(N3C(=O)c4ccc(C(c5ccc6c(c5)C(=O)N(CC(C)(C)C)C6=O)(C(F)(F)F)C(F)(F)F)cc4C3=O)cc2)c2cccc3ccccc23)cc1</smiles><smiles>CC(C)c1ccc(N(C(=O)[SnH3])c2ccc(N3C(=O)c4ccc(C(c5ccc6ccccc6c5)(c5ccc6ccccc6c5)C(F)(F)F)cc4C3=O)cc2)cc1</smiles>

Scheme 13 Chemical structures of some polymers with linkage effects. A full color version of this scheme is available at Polymer Journal online. 
that the isolated D-A system could effectively extend the retention time of the memory device. The ON/OFF current ratios of these memory devices are up to $10^{9}$. The theoretical analysis suggests that the CT mechanism could explain the memory characteristics of these PIs, and the linkage effect between the donor and the acceptor was also demonstrated.

Two PIs P55 and P56 ${ }^{94}$ were prepared from $N$-(2,4-diaminophenyl)- $N, N$-diphenylamine and $N$-(4-(2',4'-diaminophenoxy)phenyl- $N$, $\mathrm{N}$-diphenylamine with 6FDA dianhydride and were used to clarify the effects of the structure on the resulting memory properties. A memory device based on P55 showed an unstable volatile behavior, whereas a device based on P56 with bulkier donor units exhibited stable nonvolatile flash-type memory characteristics with retention times longer than $10^{4} \mathrm{~s}$. A theoretical simulation based on the DFT suggested that the greater distinct charge separation between the ground and CT states in P56 led to highly stable memory behavior. It was also confirmed that P56 had a more twisted conformation than P55 in the ground state, and the more twisted dihedral angle between the donor and acceptor units could stabilize the induced CT state, resulting in non-volatile memory characteristics.

Qi and colleagues synthesized two new diphenylnaphthylaminebased functional aromatic PIs, P57 $(\alpha \mathrm{PI})$ and P58 $(\beta \mathrm{PI})$, for memory device applications. ${ }^{95}$ By altering the naphthyl group in diphenylnaphthylamine from $\alpha$-substitution to $\beta$-substitution, the memory behaviors of the synthesized PIs can be tuned from the irreversible WORM type (P57) to the programmable flash type (P58). A P57based memory device (ITO/P57/Au) exhibits an ON/OFF current ratio as high as approximately $10^{6}$ and can be switched bi-directionally with comparable positive and negative switching-on threshold voltages, whereas the P58-based device achieves an ON/OFF ratio of up to $10^{4}$ and displays excellent reprogrammable stability during write-read-erase-reread cycles. Theoretical analysis suggests that the electric-field-induced D-A electronic transition and the subsequent formation of CT complexes could explain the electrical switching effects observed in the synthesized PIs. In addition, the more noncoplanar conformation of the diphenylnaphthylamine species in P57, compared with P58, has been suggested to bring about a higher energy barrier that prevents back CT processes under the applied electric field, leading to irreversible WORM vs programmable flash memory behaviors.

P3, which was reported by our group, exhibits DRAM behavior with a high ON/OFF current ratio of $10^{7}{ }^{52}$ However, the polyamide P4 exhibits SRAM behavior owing to the polyamide structure, which has a higher dipole moment and non-planar linkage. Both polymer memory devices revealed excellent stabilities with long operation times of $10^{4} \mathrm{~s}$ at continuously applied voltages of $-2 \mathrm{~V}$. In addition, the new functional TPA-based aromatic polyether P59-PE and polyester P59-PET (poly(ethylene terephthalate)) were synthesized for the first time for memory device applications (Scheme 14). ${ }^{96}$ For comparison, P59-PA, P59-PI and P59-PI' were also prepared to systematically investigate the relationship between chemical structure and memory behavior. The linkage effects of polyether, polyester, polyamide and PI are expected to produce different retention times in the resulting polymer memory devices owing to their different structural conformations, dipole moments, and HOMO and LUMO energy levels. P59-PE, P59-PA and P59-PET, which have non-planar linkage structures and different LUMO energy levels, show SRAM behaviors with different retention times (2, 5 and $6 \mathrm{~min}$, respectively). P59-PI with a planar linkage group exhibits DRAM behavior, whereas the corresponding isomer P59-PI' reveals insulating behavior, which could be attributed to difficulty in sustaining the CT complex. In addition, 6FPA exhibited a longer retention time than 6FPI, which
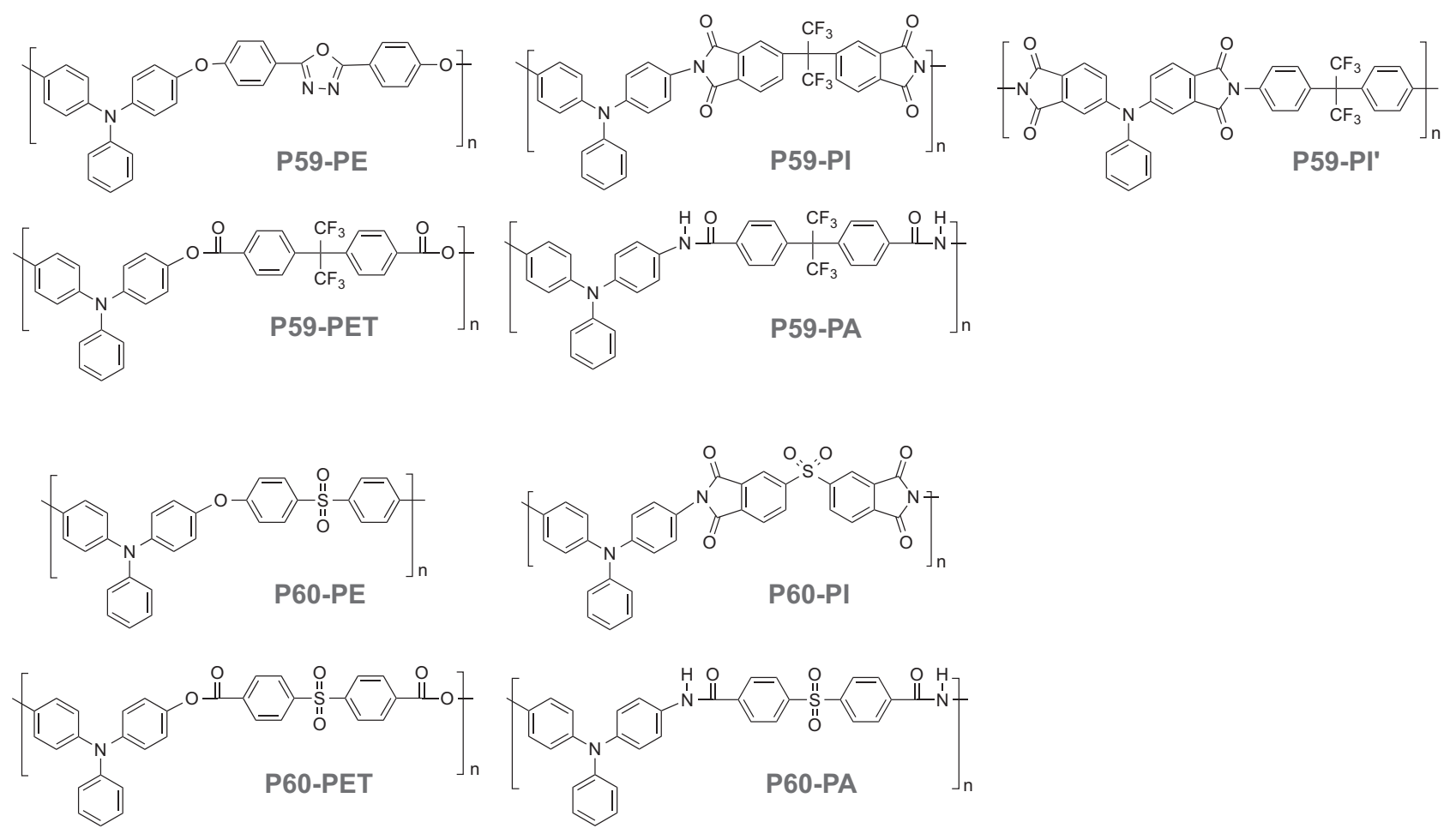

Scheme 14 Chemical structures of some polymers with linkage effects. A full color version of this scheme is available at Polymer Journal online. 
contributed to the conformation effect and higher dipole moment. The non-planar conformation of a phenyl ring with amide linkage may prevent back CT from occurring, and the higher dipole moment of 6FPA also facilitates the stabilization of the CT complex. Generally, polymers with more flexible linkages, lower LUMO energy levels, and higher dipole moments will facilitate the stability of the CT complex, and the retention time of polymer memory devices is highly dependent on the stability of the CT complex.

Our group also described the synthesis and memory properties of a series of sulfonyl-containing aromatic polymers, P60-PE, P60-PI, P60-PET and P60-PA, consisting of TPA moieties. ${ }^{97}$ P60-PE exhibited insulating behavior, whereas P60-PET, P60-PA and P60-PI exhibited SRAM behavior with retention times of 11,8 and $4 \mathrm{~min}$, respectively. By choosing the appropriate linkage between the electron donor and acceptor moieties of HPPs, tunable memory properties (from insulating to SRAM with different retention times) could be achieved.

\section{Thickness effect}

Interestingly, for polymer P61, as reported by Ree and our group (Scheme 15), film thickness can also influence memory behavior. ${ }^{98}$ Excellent WORM memory characteristics are found for films with thicknesses in the range of 34-74 nm, which may be attributed to the formation of stable local filaments. However, DRAM characteristics with ON/OFF current ratios as high as $10^{11}$ are found with film thicknesses of $100 \mathrm{~nm}$ because the films are too thick to exhibit stable local filament formation, which is responsible for the DRAM performance.
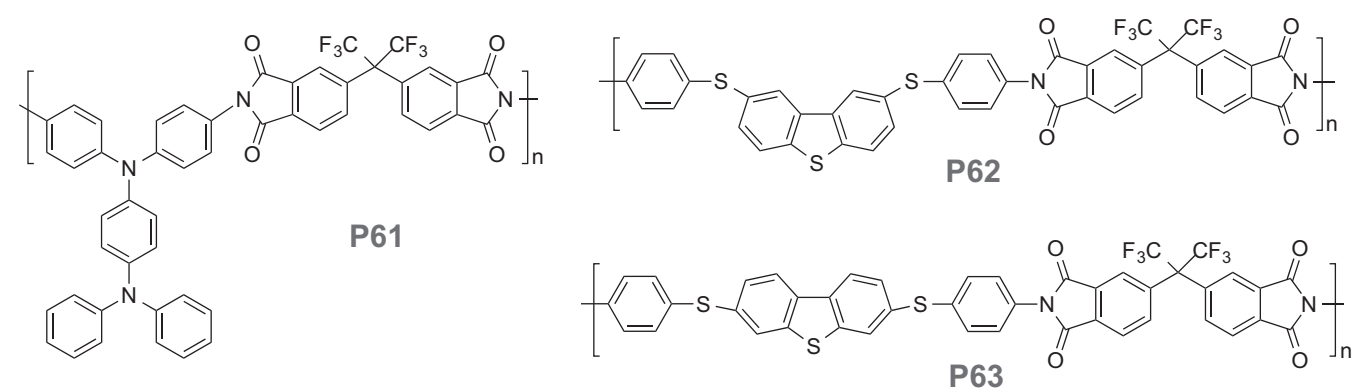

Scheme 15 Chemical structures of some polymers with thickness effects. A full color version of this scheme is available at Polymer Journal online.<smiles>Cc1ccc(N2C(=O)c3ccc(-c4ccc5c(c4)C(=O)N(C)C5=O)cc3C2=O)cc1</smiles>

P64 (BPDA/pDA Polyimide)

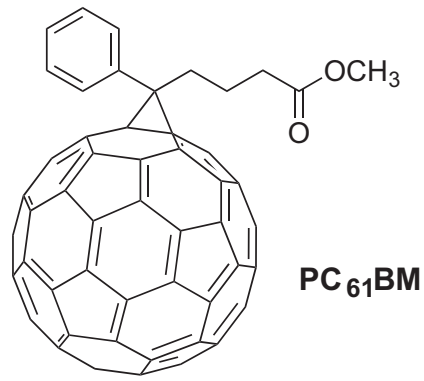<smiles>COc1ccc(N(c2ccc(C)cc2)c2ccc(C)cc2)cc1</smiles>

P65<smiles>[X]c1ccc(N(c2ccc(C(C)(C)C)cc2)c2ccc(C(C)(C)c3ccc(N(c4ccc(OC)cc4)c4ccc(C(C)(C)C)cc4)cc3)cc2)cc1</smiles>

P66

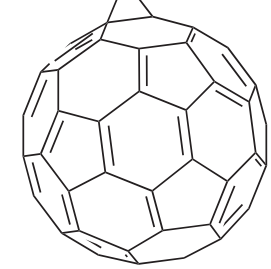

Scheme 16 Chemical structures of $\mathrm{HPP} / \mathrm{GO}$ and $\mathrm{PC}_{61} \mathrm{BM}$ hybrids. A full color version of this scheme is available at Polymer Journal online. 
In addition, Ueda and colleagues ${ }^{84}$ reported the synthesis and characterization of two PIs, P62 and P63, consisting of alternating electron-donating 2,8- or 3,7-phenylenesulfanyl-substituted dibenzothiophene and electron-accepting phthalimide moieties for high-performance memory device applications. ITO/PI/Al devices containing these PIs showed multi-memory characteristics, changing from high-conductance ohmic current flow to non-volatile negative differential resistance, DRAM and insulating, with film thicknesses of 12, 20, 25 and $45 \mathrm{~nm}$, respectively.

Our group reported that $\mathbf{P} 59-\mathbf{P E}^{96}$ exhibited insulator, SRAM and SRAM properties with thicknesses of approximately 50,40 and $30 \mathrm{~nm}$, respectively. Moreover, these TPA-based aromatic polymers exhibited not only diverse memory properties but also high ON/OFF current ratios of $10^{8}$ to $10^{9}$.

\section{HPP HYBRID MATERIALS}

Although several types of single polymer devices have been demonstrated, hybrid systems have also been extensively prepared for memory device applications. The supplementary compounds (NPs or fullerene derivatives) in these hybrid systems can be viewed as data storage media or have physical electronic transitions with the organic polymer matrix.
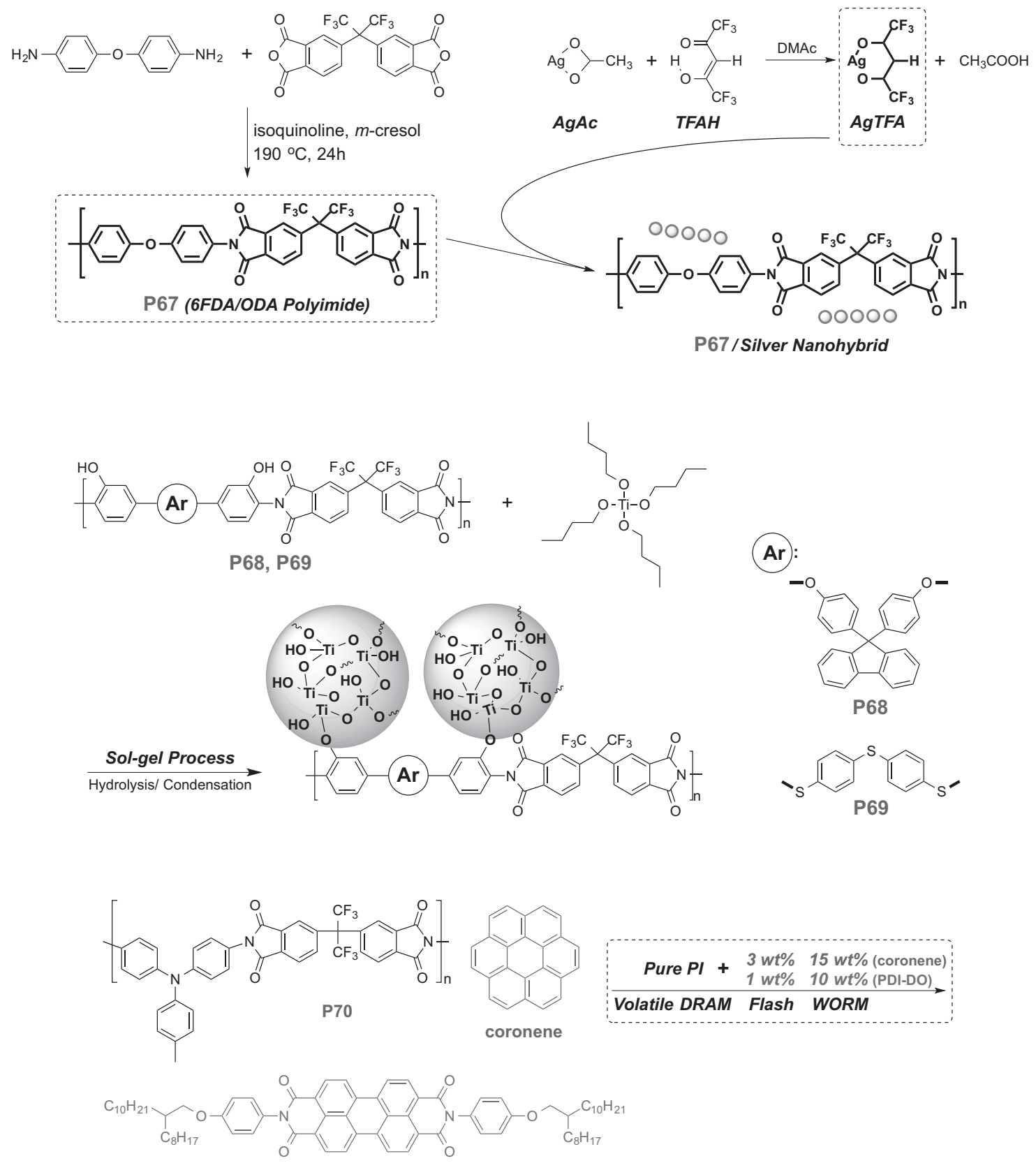

PDI-DO

Scheme 17 Chemical structures of HPP/inorganic hybrids. A full color version of this scheme is available at Polymer Journal online. 


\section{HPP/GO or $\mathrm{PC}_{61} \mathrm{BM}$ hybrids}

Lee and colleagues ${ }^{99}$ fabricated $3 \mathrm{D}$-stacked cross-bar array organic resistive memory devices with a composition of $\mathrm{P} 64: \mathrm{PC}_{61} \mathrm{BM}$, which was successfully employed as a thermally and chemically robust active organic material for stacked memory cells (Scheme 16). The fabrication yields of the operating memory cells were more than $83 \%$. The memory cells in each layer exhibited high performances with respect to non-volatile memory characteristics. This approach would enable highly integrated - and therefore more practical—organic memory devices and other organic-based electronic devices with a stackable 3D architecture to achieve increased cell density.

Furthermore, our group successfully prepared and systematically investigated a series of polymer hybrid memory materials based on thermally stable $\mathbf{P} 65$ blended with the electron acceptor $\mathrm{PC}_{61} \mathrm{BM} .{ }^{100}$ ITO/PC ${ }_{61} \mathrm{BM}: \mathrm{P} 65 / \mathrm{Al}$ memory devices exhibited both DRAM and WORM properties depending on the concentration of $\mathrm{PC}_{61} \mathrm{BM}$. The strong interaction between $\mathrm{PC}_{61} \mathrm{BM}$ and TPA that resulted in welldispersed $\mathrm{PC}_{61} \mathrm{BM}$ clusters within the $\mathrm{PC}_{61} \mathrm{BM}: \mathrm{P} 65$ hybrid films revealed bistable WORM behavior even at higher concentrations, up to $10 \mathrm{wt} \% \mathrm{PC}_{61} \mathrm{BM}$.

Moreover, our group also prepared high ON/OFF ratio, electrically bistable, non-volatile memory devices based on poly(triphenylamine) with different amounts of pendent $\mathrm{PC}_{61} \mathrm{BM}^{101}$ By introducing $10 \mathrm{wt} \%$ $\mathrm{PC}_{61} \mathrm{BM}$ into $\mathbf{P} 66$ via covalent bonding, a WORM memory device with a lower switching-ON voltage $(0.9 \mathrm{~V})$ and a much higher ON/OFF ratio $\left(10^{9}\right)$ than that of blending composites could be obtained. Moreover, the non-volatile memory behavior remained satisfactory even after heating to $100^{\circ} \mathrm{C}$ for $1 \mathrm{~h}$. This behavior is crucial for maintaining device stability in computer applications when other components produce heat.

\section{HPP/inorganic hybrids}

Tian and Qi and colleagues reported the synthesis of an electrically bistable, silver-nanoparticle-embedded PI thin film. ${ }^{102}$ A soluble PI, P67, in which the 6FDA part serves as an effective electron-accepting moiety, was synthesized as the polymer matrix (Scheme 17). Silver nanoparticles (Ag NPs) with diameters less than $7 \mathrm{~nm}$ were subsequently generated in situ in the PI film via ultraviolet (UV) reduction of a (1,1,1-trifluoro-2,4-pentadionato) silver (I) complex (AgTFA) incorporated in the matrix. Electrical characterization of the sandwiched device (ITO/PI silver nanohybrid film/Au) indicated that the nanohybrid material possessed electrical bistability and that the device exhibited non-volatile memory behavior with an ON/OFF current ratio of approximately $10^{2}$.

In addition to Ag NPs, some other types of inorganic nanomaterials have been introduced into polymers to realize rewritable memory behavior. We have synthesized novel solution-processable sulfur- or fluorene-containing poly(o-hydroxyimide) $\mathbf{P 6 8} \mathbf{8}^{103}$ and $\mathbf{P 6 9} 9^{104}$ and their corresponding $\mathrm{PI} / \mathrm{TiO}_{2}$ hybrids used them for memory applications. To adjust the memory behavior, different amounts of $\mathrm{TiO}_{2}$ were introduced into the PIs, and the corresponding tunable memory properties were investigated. The pendant hydroxyl groups on the backbone of PIs can provide reaction sites for organic-inorganic bonding and the homogeneous hybrid thin films can therefore be obtained by controlling the mole ratio of titanium butoxide/hydroxyl groups via sol-gel reaction. The resulting hybrid films with different $\mathrm{TiO}_{2}$ concentrations from 0 wt $\%$ to 50 wt $\%$ exhibited electrically programmable digital memory properties from DRAM to SRAM and WORM with a high ON/OFF current ratio of $10^{8}$. Furthermore, from the results of the $I-V$ characteristics, the crystalline phase of titania has a higher trapping ability, increasing the retention time of the ON state.
Other resistive switching memory devices based on PI blends have been described by Ueda and colleagues. ${ }^{105}$ The active layers of the PI blend films were prepared from different compositions of P70 and polycyclic aromatic compounds (coronene or N,N-bis[4(2-octyldodecyloxy)phenyl]-3,4,9,10-perylenetetracarboxylic diimide (PDI-DO)). The addition of large $\pi$-conjugated polycyclic compounds can stabilize the CT complex induced by the applied electric field. Thus, the memory device behavior changes from the volatile to nonvolatile characteristics of flash and WORM, respectively, as the additive contents increase in both blend systems. The main differences between these two blend systems are the threshold voltage values and the additive contents needed to obtain the memory properties. Owing to the stronger accepting ability and higher electron affinity of PDIDO than coronene, P70:PDI-DO blend-based memory devices show lower threshold voltages, and the memory behavior could be tuned by controlling the additive concentration.

\section{FLEXIBLE HPP ELECTRICAL MEMORY DEVICES}

Like other organic electronics, flexible polymer memory devices using PI as an active layer have been demonstrated. ${ }^{53,68,106}$ Ueda and colleagues fabricated a typical memory device on a flexible PET substrate. ${ }^{68}$ The device showed very stable non-volatile behavior even after bending at a radius of curvature of $5 \mathrm{~mm}$ for up to 1000 bending cycles.

Additionally, the flexible memory device reported by our group ${ }^{106}$ was tested under severe bending at various radii of curvature including $11,9,7$ and $5 \mathrm{~mm}$, and the flexible device did not crack or deform upon bending. The reliable and reproducible switching memory behavior of the PI film in the device could also be obtained under mechanical bending stress. The development of device fabrication techniques for practical use has been performed similar to those of other organic electronics, such as organic transistors and organic photovoltaics, and the performance of polymer memory devices should depend significantly on the fabrication process. Therefore, similar to the development of the memory material, there is still significant potential in this area.

\section{CONCLUSIONS AND PERSPECTIVES}

The development of polymer materials for application as low-cost, high-mechanical-strength, solution-processable and high-density data storage memory devices is an emerging area. Considering heat resistance during the device fabrication process and operation, HPPs are the most attractive candidates for memory applications owing to their high thermal/dimensional stabilities and mechanical strengths. In this review, we have summarized the most widely used mechanisms in HPP resistive memory devices, such as CT, space charge trapping, and filament conduction. In addition, energetic studies around the world have shown the great potential of HPPs as memory device materials. By carefully designing the polymeric structure based on these systematically investigated switching mechanisms, almost all types of current memory characteristics can be reproduced, and these memory properties show extremely high endurance during long-term operation, which again makes HPPs, such as polyimide, very suitable materials for memory applications.

\section{CONFLICT OF INTEREST}

The authors declare no conflict of interest.

\section{ACKNOWLEDGEMENTS}

We gratefully acknowledge the Ministry of Science and Technology of Taiwan for financial support. 
1 Hill, R. \& Walker, E. E. Polymer constitution and fiber properties. J. Polym. Sci. 3, 609-630 (1948).

2 Bell, V. L., Stump, B. L. \& Gager, H. Polyimide structure-property relationships. II. Polymers from isomeric diamines. J. Polym. Sci. Polym. Chem. Ed. 14 , 2275-2291 (1976).

3 Cassidy, P. E. Thermally Stable Polymers: Synthesis and Properties (Marcel Dekker, New York, 1980).

4 Clair, T. L. S. \& Yamaki, D. A. Solvent resistant, thermoplastic aromatic poly (imidesulfone) and process for preparing same. U. S. Patent 4,398,021 (1983).

5 Clair, T. L. S. \& Yamaki, D. A. Process for preparing solvent resistant, thermoplastic aromatic poly(imidesulfone). U. S. patent 4,489,027 (1984).

6 Hergenrother, P. M. High performance thermoplastics. Die Angew Makromol. Chem. 145, 323-341 (1986)

7 Wilson, D., Stenzenberger, H. D. \& Hergenrother, P. M. Polyimides (Springer: Netherlands, 1990).

8 Yang, H. Kevlar Aramid Fiber (John Wiley \& Sons, New York, 1993).

9 Ghosh, M. K. \& Mittal, K. L. Polyimides: Fundamentals and Applications (Marcel Dekker, New York, 1996).

10 de Abajo, J. \& de la Campa, J. G. in Progress in Polyimide Chemistry I Vol. 140 Advances in Polymer Science (ed. Kricheldorf H. R.) Ch. 2, 23-59 (Springer, Berlin Heidelberg, 1999).

11 Mittal, K. L. Polyimides: Synthesis, Characterization, and Applications. Vol. 1 (Plenum, New York, 1984).

12 Yang, H. H. Aromatic High-Strength Fibers (John Wiley \& Sons, Inc., New York, 1989).

13 Abadie, M. J. M. \& Mittal, B. Polyimides and Other High Temperature Polymers (Elsevier, Amsterdam, 1991).

14 Cassidy, P. E. Thermally Stable Polymers (Marcel Dekker, New York, 1980).

15 Imai, Y. Synthesis of novel organic-soluble high-temperature aromatic polymers. High Perform. Polym. 7, 337-345 (1995).

16 Imai, Y. Recent advances in synthesis of high-temperature aromatic polymers. React. Funct. Polym. 30, 3-15 (1996).

17 Hsiao, S.-H. \& Li, C.-T. Synthesis and characterization of new adamantane-based polyimides. Macromolecules 31, 7213-7217 (1998).

18 Liou, G.-S. Synthesis and properties of soluble aromatic polyimides from 2,2'-bis(3,4dicarboxyphenoxy)-1,1'-binaphthyl dianhydride and aromatic diamines. J. Polym. Sci. A Polym. Chem. 36, 1937-1943 (1998).

19 Eastmond, G. C., Gibas, M. \& Paprotny, J. Pendant adamantyl poly(ether imide)s: synthesis and a preliminary study of properties. Eur. Polym. J. 35 , 2097-2106 (1999)

20 Eastmond, G. C., Paprotny, J. \& Irwin, R. S. 1,2-Bis(carboxyphenoxy)arylenes and aramids and polyarylates therefrom: synthesis and properties. Polymer 40, 469-486 (1999).

21 Sahadeva Reddy, D., Chou, C.-H., Shu, C.-F. \& Lee, G.-H. Synthesis and characterization of soluble poly(ether imide)s based on 2,2'-bis(4-aminophenoxy)-9,9'-spirobifluorene. Polymer 44, 557-563 (2003).

22 Myung, B. Y., Ahn, C. J. \& Yoon, T. H. Synthesis and characterization of polyimides from novel 1-(3',5'-bis(trifluoromethyl)benzene) pyromelliticdianhydride (6FPPMDA). Polymer 45, 3185-3193 (2004).

23 Liou, G.-S., Hsiao, S.-H., Ishida, M., Kakimoto, M. \& Imai, Y. Synthesis and characterization of novel soluble triphenylamine-containing aromatic polyamides based on $N, N$ '-bis(4-aminophenyl)- $N, N^{\prime}$-diphenyl-1,4-phenylenediamine. J. Polym. Sci. A Polym. Chem. 40, 2810-2818 (2002).

24 Liou, G.-S. \& Hsiao, S.-H. Synthesis and properties of new soluble triphenylaminebased aromatic poly(amine amide)s derived from $N, N^{\prime}$-bis(4-carboxyphenyl)- $N, N^{\prime}$ diphenyl-1,4-phenylenediamine. J. Polym. Sci. A Polym. Chem. 41, 94-105 (2003).

25 Hsiao, S.-H., Chen, C.-W. \& Liou, G.-S. Novel aromatic polyamides bearing pendent diphenylamino or carbazolyl groups. J. Polym. Sci. A Polym. Chem. 42, 3302-3313 (2004).

26 Oishi, Y., Ishida, M., Kakimoto, M.-A., Imai, Y. \& Kurosaki, T. Preparation and properties of novel soluble aromatic polyimides from 4,4'-diaminotriphenylamine and aromatic tetracarboxylic dianhydrides. J. Polym. Sci. A Polym. Chem. 30, 1027-1035 (1992)

27 Okamoto, K., Tanaka, K., Kita, H., Ishida, M., Kakimoto, M. \& Imai, Y. Gas permeability and permselectivity of polyimides prepared from $4,4^{\prime}$-diaminotriphenylamine. Polym. J. 24, 451-457 (1992).

28 Oishi, Y., Takado, H., Yoneyama, M., Kakimoto, M.-A. \& Imai, Y. Preparation and properties of new aromatic polyamides from 4,4'-diaminotriphenylamine and aromatic dicarboxylic acids. J. Polym. Sci. A Polym. Chem. 28, 1763-1769 (1990).

29 Murofushi, Y., Ishikawa, M. \& Kawai, M. Polymer of triphenylamine. U. S. patent 4,565,860 (1986)

30 Goodson, F. E., Hauck, S. I. \& Hartwig, J. F. Palladium-catalyzed synthesis of pure, regiodefined polymeric triarylamines. J. Am. Chem. Soc. 121, 7527-7539 (1999).

31 Ogino, K., Kanegae, A., Yamaguchi, R., Sato, H. \& Kurjata, J. Oxidative coupling polymerization of 4-methyltriphenylamine. Macromol. Rapid Commun. 20, 103-106 (1999).

32 Lin, H.-Y. \& Liou, G.-S. Poly(triphenylamine)s derived from oxidative coupling reaction: substituent effects on the polymerization, electrochemical, and electrooptical properties. J. Polym. Sci. A Polym. Chem. 47, 285-294 (2009).
33 Ling, Q.-D., Liaw, D.-J., Zhu, C., Chan, D. S.-H., Kang, E.-T. \& Neoh, K.-G. Polymer electronic memories: Materials, devices and mechanisms. Prog. Polym. Sci. 33, 917-978 (2008)

34 You, N.-H., Chueh, C.-C., Liu, C.-L., Ueda, M. \& Chen, W.-C. Synthesis and memory device characteristics of new sulfur donor containing polyimides. Macromolecules 42, 4456-4463 (2009)

35 Smits, J. H. A., Meskers, S. C. J., Janssen, R. A. J., Marsman, A. W. \& de Leeuw, D. M. Electrically rewritable memory cells from poly(3-hexylthiophene) Schottky diodes. Adv. Mater. 17, 1169-1173 (2005).

36 Tang, W., Shi, H. Z., Xu, G., Ong, B. S., Popovic, Z. D., Deng, J. C., Zhao, J. \& Rao, G. H. Memory effect and negative differential resistance by electrode-induced two-dimensional single-electron tunneling in molecular and organic electronic devices. Adv. Mater. 17, 2307-2311 (2005).

37 Verbakel, F., Meskers, S. C. J. \& Janssen, R. A. J. Electronic memory effects in a sexithiophene-poly(ethylene oxide) block copolymer doped with $\mathrm{NaCl}$. Combined diode and resistive switching behavior. Chem. Mater. 18, 2707-2712 (2006).

38 Lin, H.-T., Pei, Z., Chen, J.-R., Hwang, G.-W., Fan, J.-F. \& Chan, Y.-J. A new nonvolatile bistable polymer-nanoparticle memory device. IEEE Electr Device Lett. $\mathbf{2 8}$ 951-953 (2007).

39 Attianese, D., Petrosino, M., Vacca, P., Concilio, S., lannelli, P., Rubino, A. \& Bellone, S. Switching device based on a thin film of an azo-containing polymer for application in memory cells. IEEE Electr Device Lett. 29, 44-46 (2008).

40 Cho, B., Kim, T.-W., Choe, M., Wang, G., Song, S. \& Lee, T. Unipolar nonvolatile memory devices with composites of poly(9-vinylcarbazole) and titanium dioxide nanoparticles. Org. Electron. 10, 473-477 (2009).

41 Zhuang, X.-D., Chen, Y., Liu, G., Zhang, B., Neoh, K.-G., Kang, E.-T., Zhu, C.-X., Li, Y.-X. \& Niu, L.-J. Preparation and memory performance of a nanoaggregated dispersed red 1-functionalized poly ( $N$-vinylcarbazole) film via solution-phase self-assembly. Adv. Funct. Mater. 20, 2916-2922 (2010).

42 Chu, C. W., Ouyang, J., Tseng, H. H. \& Yang, Y. Organic donor-acceptor system exhibiting electrical bistability for use in memory devices. Adv. Mater. 17, 1440-1443 (2005)

43 Ling, Q.-D., Chang, F.-C., Song, Y., Zhu, C.-X., Liaw, D.-J., Chan, D. S.-H., Kang, E.-T. \& Neoh, K.-G. Synthesis and dynamic random access memory behavior of a functional polyimide. J. Am. Chem. Soc. 128, 8732-8733 (2006).

44 Lai, Y.-C., Ohshimizu, K., Lee, W.-Y., Hsu, J.-C., Higashihara, T., Ueda, M. \& Chen, W.-C. Electrically bistable memory devices based on all-conjugated block copolythiophenes and their PCBM composite films. J. Mater. Chem. 21, 14502-14508 (2011).

45 Li, Y.-Q., Fang, R.-C., Zheng, A.-M., Chu, Y.-Y., Tao, X., Xu, H.-H., Ding, S.-J. \& Shen, Y.-Z. Nonvolatile memory devices based on polyimides bearing noncoplanar twisted biphenyl units containing carbazole and triphenylamine side-chain groups. J. Mater. Chem. 21, 15643-15654 (2011).

46 Zhang, B., Liu, Y.-L., Chen, Y., Neoh, K.-G., Li, Y.-X., Zhu, C.-X., Tok, E.-S. \& Kang, E.-T. Nonvolatile rewritable memory effects in graphene oxide functionalized by conjugated polymer containing fluorene and carbazole units. Chemistry 17, 10304-10311 (2011).

47 Liu, S.-J., Lin, Z.-H., Zhao, Q., Ma, Y., Shi, H.-F., Yi, M.-D., Ling, Q.-D., Fan, Q.-L., Zhu, C.-X., Kang, E.-T. \& Huang, W. Flash-memory effect for polyfluorenes with onchain Iridium(III) complexes. Adv. Funct. Mater. 21, 979-985 (2011).

48 Chen, C.-J., Yen, H.-J., Chen, W.-C. \& Liou, G.-S. Resistive switching non-volatile and volatile memory behavior of aromatic polyimides with various electron-withdrawing moieties. J. Mater. Chem. 22, 14085-14093 (2012).

49 Zhang, B., Liu, G., Chen, Y., Wang, C., Neoh, K.-G., Bai, T. \& Kang, E-T. Electrical bistability and WORM memory effects in donor-acceptor polymers based on poly $(\mathrm{N}$ vinylcarbazole). Chempluschem. 77, 74-81 (2012).

50 Liu, C.-L. \& Chen, W.-C. Donor-acceptor polymers for advanced memory device applications. Polym. Chem. 2, 2169-2174 (2011).

51 Kuorosawa, T., Chueh, C.-C., Liu, C.-L., Higashihara, T., Ueda, M. \& Chen, W.-C. High performance volatile polymeric memory devices based on novel triphenylamine-based polyimides containing mono- or dual-mediated phenoxy linkages. Macromolecules $\mathbf{4 3}$, 1236-1244 (2010)

52 Chen, C.-J., Yen, H.-J., Chen, W.-C. \& Liou, G.-S. Novel high-performance polymer memory devices containing $(\mathrm{OMe}) 2$ tetraphenyl-p-phenylenediamine moieties. J. Polym. Sci. A Polym. Chem. 49, 3709-3718 (2011).

53 Chen, C.-J., Yen, H.-J., Hu, Y.-C. \& Liou, G.-S. Novel programmable functional polyimides: preparation, mechanism of CT induced memory, and ambipolar electrochromic behavior. J. Mater. Chem. C 1, 7623-7634 (2013).

54 Kurosawa, T., Lai, Y.-C., Higashihara, T., Ueda, M., Liu, C.-L. \& Chen, W.-C. Tuning the electrical memory characteristics from volatile to nonvolatile by perylene Imide composition in random copolyimides. Macromolecules 45, 4556-4563 (2012).

55 Carchano, H., Lacoste, R. \& Segui, Y. Bistable electrical switching in polymer thin films. Appl. Phys. Lett. 19, 414-415 (1971).

56 Pender, L. F. \& Fleming, R. J. Memory switching in glow-discharge polymerized thinfilms. J. Appl. Phys. 46, 3426-3431 (1975).

57 Segui, Y., Ai, B. \& Carchano, H. Switching in polystyrene films: Transition from ON to OFF state. J. Appl. Phys. 47, 140-143 (1976).

58 Hwang, W. \& Kao, K. C. Theory of filamentary double injection and electroluminescence in molecular-crystals. J. Chem. Phys. 60, 3845-3855 (1974).

59 Wierschem, A., Niedernostheide, F. J., Gorbatyuk, A. \& Purwins, H. G. Observation of current-density filamentation in multilayer structures by EBIC measurements. Scanning 17, 106-116 (1995). 
60 Joo, W.-J., Choi, T.-L., Lee, J., Lee, S. K., Jung, M.-S., Kim, N. \& Kim, J. M. Metal filament growth in electrically conductive polymers for nonvolatile memory application. J. Phys. Chem. B 110, 23812-23816 (2006)

61 Liu, Y.-L., Ling, Q.-D., Kang, E.-T., Neoh, K.-G., Liaw, D.-J., Wang, K.-L., Liou, W.-T., Zhu, C.-X. \& Chan, D. S.-H. Volatile electrical switching in a functional polyimide containing electron-donor and -acceptor moieties. J. Appl. Phys. 105, 044501 (2009).

62 Tian, G., Wu, D., Qi, S., Wu, Z. \& Wang, X. Dynamic random access memory effect and memory device derived from a functional polyimide containing electron donor-acceptor pairs in the main chain. Macromol. Rapid Commun. 32, 384-389 (2011)

63 Liu, Y.-L., Wang, K.-L., Huang, G.-S., Zhu, C.-X., Tok, E.-S., Neoh, K.-G. \& Kang, E.-T. Volatile electrical switching and static random access memory effect in a functional polyimide containing oxadiazole moieties. Chem. Mater. 21, 3391-3399 (2009).

64 Liu, Y., Zhang, Y., Lan, Q., Liu, S., Qin, Z., Chen, L., Zhao, C., Chi, Z. \& Xu, J. \& Economy, J. High-performance functional polyimides containing rigid nonplanar conjugated triphenylethylene moieties. Chem. Mater. 24, 1212-1222 (2012)

65 Liu, Y., Zhang, Y., Lan, Q., Qin, Z., Liu, S., Zhao, C., Chi, Z. \& Xu, J. Synthesis and properties of high-performance functional polyimides containing rigid nonplanar conjugated tetraphenylethylene moieties. J. Polym. Sci. A Polym. Chem. 51 1302-1314 (2013)

66 Liou, G.-S., Hsiao, S.-H. \& Chen, H.-W. Novel high-Tg poly(amine-imide)s bearing pendent $\mathrm{N}$-phenylcarbazole units: synthesis and photophysical, electrochemical and electrochromic properties. J. Mater. Chem. 16, 1831-1842 (2006).

67 Shi, L., Tian, G., Ye, H., Qi, S. \& Wu, D. Volatile static random access memory behavior of an aromatic polyimide bearing carbazole-tethered triphenylamine moieties. Polymer 55, 1150-1159 (2014).

68 Yu, A.-D., Kurosawa, T., Lai, Y.-C., Higashihara, T., Ueda, M., Liu, C.-L. \& Chen, W.-C. Flexible polymer memory devices derived from triphenylamine-pyrene containing donor-acceptor polyimides. J. Mater. Chem. 22, 20754-20763 (2012).

69 Kim, D. M., Park, S., Lee, T. J., Hahm, S. G., Kim, K., Kim, J. C., Kwon, W. \& Ree, M. Programmable permanent data storage characteristics of nanoscale thin films of a thermally stable aromatic polyimide. Langmuir. 25, 11713-11719 (2009).

70 Liu, Q., Jiang, K., Wen, Y., Wang, J., Luo, J. \& Song, Y. High-performance optoelectrical dual-mode memory based on spiropyran-containing polyimide. Appl. Phys. Lett. 97, 253304 (2010).

71 Wang, K.-L., Liu, Y.-L., Lee, J.-W., Neoh, K.-G. \& Kang, E.-T. Nonvolatile electrical switching and write-once read-many-times memory effects in functional polyimides containing triphenylamine and 1,3,4-oxadiazole moieties. Macromolecules 43, 7159-7164 (2010)

72 Wang, K. L., Liu, Y. L., Shih, I. H., Neoh, K. G. \& Kang, E. T. Synthesis of polyimides containing triphenylamine-substituted triazole moieties for polymer memory applications. J. Polym. Sci. A Polym. Chem. 48, 5790-5800 (2010).

73 Park, S., Kim, K., Kim, J. C., Kwon, W., Kim, D. M. \& Ree, M. Synthesis and nonvolatile memory characteristics of thermally, dimensionally and chemically stable polyimides. Polymer 52, 2170-2179 (2011).

74 Chen, F., Tian, G., Shi, L., Qi, S. \& Wu, D. Nonvolatile write-once read-many-times memory device based on an aromatic hyperbranched polyimide bearing triphenylamine moieties. RSC Advances 2, 12879-12885 (2012).

75 Hahm, S. G., Choi, S., Hong, S.-H., Lee, T. J., Park, S., Kim, D. M., Kim, J. C., Kwon, W., Kim, K., Kim, M.-J., Kim, O. \& Ree, M. Electrically bistable nonvolatile switching devices fabricated with a high performance polyimide bearing diphenylcarbamyl moieties. J. Mater. Chem. 19, 2207-2214 (2009).

76 Kim, K., Park, S., Hahm, S. G., Lee, T. J., Kim, D. M., Kim, J. C., Kwon, W., Ko, Y.-G. \& Ree, M. Nonvolatile unipolar and bipolar bistable memory characteristics of a high temperature polyimide bearing diphenylaminobenzylidenylimine moieties. J. Phys. Chem. B 113, 9143-9150 (2009).

77 Hu, B., Zhuge, F., Zhu, X., Peng, S., Chen, X., Pan, L., Yan, Q. \& Li, R.-W. Nonvolatile bistable resistive switching in a new polyimide bearing 9-phenyl-9H-carbazole pendant. J. Mater. Chem. 22, 520-526 (2012).

78 Hahm, S. G., Choi, S., Hong, S.-H., Lee, T. J., Park, S., Kim, D. M., Kwon, W.-S., Kim, K., Kim, O. \& Ree, M. Novel rewritable, non-volatile memory devices based on thermally and dimensionally stable polyimide thin films. Adv. Funct. Mater. 18 3276-3282 (2008)

79 Park, S., Kim, K., Kim, D. M., Kwon, W., Choi, J. \& Ree, M. High temperature polyimide containing anthracene moiety and its structure, interface, and nonvolatile memory behavior. ACS Appl. Mater. Interfaces 3, 765-773 (2011).

$80 \mathrm{Li}$, Y., Fang, R., Ding, S. \& Shen, Y. Rewritable and non-volatile memory effects based on polyimides containing pendant carbazole and triphenylamine groups. Macromol. Chem. Phys. 212, 2360-2370 (2011).

81 Tian, G., Qi, S., Chen, F., Shi, L., Hu, W. \& Wu, D. Nonvolatile memory effect of a functional polyimide containing ferrocene as the electroactive moiety. Appl. Phys. Lett. 98, 203302 (2011).

82 Liu, S.-H., Yang, W.-L., Wu, C.-C., Chao, T.-S., Ye, M.-R., Su, Y.-Y., Wang, P.-Y. \& Tsai, M.-J. High-performance polyimide-based ReRAM for nonvolatile memory application. IEEE Electr. Dev. Lett. 34, 123-125 (2013).

83 Lee, T. J., Park, S., Hahm, S. G., Kim, D. M., Kim, K., Kim, J., Kwon, W., Kim, Y., Chang, T. \& Ree, M. Programmable digital memory characteristics of nanoscale thin films of a fully conjugated polymer. J. Phys. Chem. C 113, 3855-3861 (2009).

84 Liu, C.-L., Kurosawa, T., Yu, A.-D., Higashihara, T., Ueda, M. \& Chen, W.-C. New dibenzothiophene-containing donor-acceptor polyimides for high-performance memory device applications. J. Phys. Chem. C 115, 5930-5939 (2011).

85 Lee, T. J., Ko, Y.-G., Yen, H.-J., Kim, K., Kim, D. M., Kwon, W., Hahm, S. G., Liou, G.-S. \& Ree, M. Programmable digital nonvolatile memory behaviors of donoracceptor polyimides bearing triphenylamine derivatives: effects of substituents. Polym. Chem. 3, 1276-1283 (2012).

86 Li, Y., Xu, H., Tao, X., Qian, K., Fu, S., Shen, Y. \& Ding, S. Synthesis and memory characteristics of highly organo-soluble polyimides bearing a noncoplanar twisted biphenyl unit containing aromatic side-chain groups. J. Mater. Chem. 21, 1810-1821 (2011).

87 Li, Y., Chu, Y., Fang, R., Ding, S., Wang, Y., Shen, Y. \& Zheng, A. Synthesis and memory characteristics of polyimides containing noncoplanar aryl pendant groups. Polymer 53, 229-240 (2012).

88 Kim, D. M., Ko, Y.-G., Choi, J. K., Kim, K., Kwon, W., Jung, J., Yoon, T.-H. \& Ree, M. Digital memory behaviors of aromatic polyimides bearing bis(trifluoromethyl)- and bithiophenyl-triphenylamine units. Polymer 53, 1703-1710 (2012).

89 Kim, K., Yen, H.-J., Ko, Y.-G., Chang, C.-W., Kwon, W., Liou, G.-S. \& Ree, M. Electrically bistable digital memory behaviors of thin films of polyimides based on conjugated bis(triphenylamine) derivatives. Polymer 53, 4135-4144 (2012).

90 Ko, Y.-G., Kwon, W., Yen, H.-J., Chang, C.-W., Kim, D. M., Kim, K., Hahm, S. G. Lee, T. J., Liou, G.-S. \& Ree, M. Various digital memory behaviors of functional aromatic polyimides based on electron donor and acceptor substituted triphenylamines. Macromolecules 45, 3749-3758 (2012).

91 Li, Y., Xu, H., Tao, X., Qian, K., Fu, S., Ding, S. \& Shen, Y. Resistive switching characteristics of polyimides derived from 2,2'-aryl substituents tetracarboxylic dianhydrides. Polymer Int. 60, 1679-1687 (2011).

92 Kurosawa, T., Lai, Y.-C., Yu, A.-D., Wu, H.-C., Higashihara, T., Ueda, M \& Chen, W.-C. Effects of the acceptor conjugation length and composition on the electrical memory characteristics of random copolyimides. J. Polym. Sci. A Polym. Chem. 51, 1348-1358 (2013).

93 Hu, Y.-C., Chen, C.-J., Yen, H.-J., Lin, K.-Y., Yeh, J.-M., Chen, W.-C. \& Liou, G.-S. Novel triphenylamine-containing ambipolar polyimides with pendant anthraquinone moiety for polymeric memory device, electrochromic and gas separation applications. J. Mater. Chem. 22, 20394-20402 (2012).

94 Kurosawa, T., Yu, A.-D., Higashihara, T., Chen, W.-C. \& Ueda, M. Inducing a high twisted conformation in the polyimide structure by bulky donor moieties for the development of non-volatile memory. Eur. Polym. J. 49, 3377-3386 (2013)

95 Shi, L., Ye, H., Liu, W., Tian, G., Qi, S. \& Wu, D. Tuning the electrical memory characteristics from WORM to flash by alpha- and beta-substitution of the electrondonating naphthylamine moieties in functional polyimides. J. Mater. Chem. C 1, 7387-7399 (2013)

96 Chen, C.-J., Hu, Y.-C. \& Liou, G.-S. Linkage and acceptor effects on diverse memory behavior of triphenylamine-based aromatic polymers. Polym. Chem. 4 , 4162-4171 (2013).

97 Chen, C.-J., Hu, Y.-C. \& Liou, G.-S. Linkage effect on the memory behavior of sulfonylcontaining aromatic polyether, polyester, polyamide, and polyimide. Chem. Commun. 49, 2536-2538 (2013)

98 Lee, T. J., Chang, C.-W., Hahm, S. G., Kim, K., Park, S., Kim, D. M., Kim, J., Kwon, W.-S., Liou, G.-S. \& Ree, M. Programmable digital memory devices based on nanoscale thin films of a thermally dimensionally stable polyimide. Nanotechnology 20, 135204 (2009).

99 Song, S., Cho, B., Kim, T.-W., Ji, Y., Jo, M., Wang, G., Choe, M., Kahng, Y. H., Hwang, H. \& Lee, T. Three-dimensional integration of organic resistive memory devices. Adv. Mater. 22, 5048-5052 (2010).

100 Chen, C.-J., Hu, Y.-C. \& Liou, G.-S. Electrically bistable memory devices based on poly(triphenylamine)-PCBM hybrids. Chem. Commun. 49, 2804-2806 (2013).

101 Chen, C.-J., Wu, J.-H. \& Liou, G.-S. Thermally stable and high ON/OFF ratio nonvolatile memory devices based on poly(triphenylamine) with pendent PCBM. Chem. Commun. 50, 4335-4337 (2014).

102 Tian, G., Wu, D., Shi, L., Qi, S. \& Wu, Z. Nonvolatile electrical switching behavior observed in a functional polyimide thin film embedded with silver nanoparticles. RSC Advances 2, 9846-9850 (2012)

103 Tsai, C.-L., Chen, C.-J., Wang, P.-H., Lin, J.-J. \& Liou, G.-S. Novel solutionprocessable fluorene-based polyimide/TiO2 hybrids with tunable memory properties. Polym. Chem. 4, 4570-4573 (2013).

104 Chen, C.-J., Tsai, C.-L. \& Liou, G.-S. Electrically programmable digital memory behaviors based on novel functional aromatic polyimide/TiO2 hybrids with a high ON/OFF ratio. J. Mater. Chem. C 2, 2842-2850 (2014).

105 Yu, A.-D., Kurosawa, T., Chou, Y.-H., Aoyagi, K., Shoji, Y., Higashihara, T., Ueda, M., Liu, C.-L. \& Chen, W.-C. Tunable electrical memory characteristics using polyimide: Polycyclic aromatic compound blends on flexible substrates. ACS Appl. Mater. Interfaces 5, 4921-4929 (2013).

106 Yen, H.-J., Chen, C.-J. \& Liou, G.-S. Flexible multi-colored electrochromic and volatile polymer memory devices derived from starburst triarylamine-based electroactive polyimide. Adv. Funct. Mater. 23, 5307-5316 (2013). 


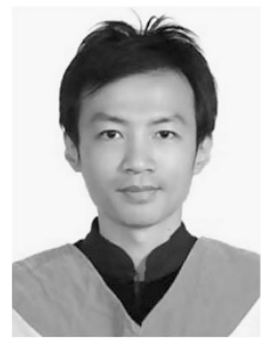

Hung-Ju Yen received his Ph.D. degree with the 1st prize of PhD graduates from Institute of Polymer Science and Engineering in 2011 under the guidance of Prof. Guey-Sheng Liou at National Taiwan University (NTU). He joined Los Alamos National Laboratory as a J. Robert Oppenheimer Distinguished Postdoctoral Fellow after working as a postdoctoral researcher at NTU for a year. He has written/ involved in several grant proposals funded by Los Alamos Directed Research and Development grant, Ministry of Science and Technology of Taiwan, Ministry of Economic Affairs, Industrial Technology Research Institute, and Institute of Nuclear Energy Research. His current research interest is in the bottom-up synthesized nanographenes and organic-inorganic perovskites for organic electronic and energy devices.

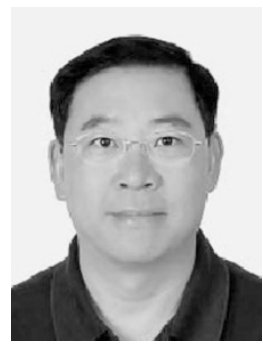

Guey-Sheng Liou received his Ph.D. degree from Department of Organic and Polymeric Materials in 1993 under the supervision of Prof. Yoshio Imai at Tokyo Institute of Technology. He then worked as a researcher in Union Chemical Laboratory, Industrial Technology and Research Institute, Taiwan, until July 1996. He joined I-Shou University and was promoted to professor in 2000. He moved to National Chi Nan University during 2001 to 2007. He is presently a distinguished professor of Institute of Polymer Science and Engineering, National Taiwan University. His research interests include the development of organic electrochromic materials, lightemitting aromatic polymers, thermally stable polymers for microelectronics and energy-related applications and polymer-inorganic hybrid materials for optical and electronic applications. 